Article

\title{
Synergistic Adverse Effects of Azithromycin and Hydroxychloroquine on Human Cardiomyocytes at a Clinically Relevant Treatment Duration
}

\author{
Wener Li ${ }^{1,+}\left(\mathbb{D}\right.$, Xiaojing Luo ${ }^{1,+}$, Mareike S. Poetsch ${ }^{1}$, Reinhard Oertel ${ }^{2}$, Kapil Nichani ${ }^{3}$, Martin Schneider ${ }^{3}$, \\ Anna Strano ${ }^{1}$, Marcel Hasse ${ }^{1}$, Robert-Patrick Steiner ${ }^{1}$, Lukas Cyganek ${ }^{4,5}$ (D), Karina Hettwer ${ }^{3}$, Steffen Uhlig ${ }^{3}$, \\ Kirsten Simon ${ }^{3}$, Kaomei Guan ${ }^{1, *, \ddagger(D)}$ and Mario Schubert ${ }^{1, *, \ddagger}$
}

check for updates

Citation: Li, W.; Luo, X.; Poetsch, M.S.; Oertel, R.; Nichani, K.;

Schneider, M.; Strano, A.; Hasse, M.; Steiner, R.-P.; Cyganek, L.; et al. Synergistic Adverse Effects of Azithromycin and

Hydroxychloroquine on Human Cardiomyocytes at a Clinically

Relevant Treatment Duration.

Pharmaceuticals 2022, 15, 220.

https://doi.org/10.3390/

ph15020220

Academic Editor: Saima Imran

Received: 20 January 2022

Accepted: 10 February 2022

Published: 12 February 2022

Publisher's Note: MDPI stays neutral with regard to jurisdictional claims in published maps and institutional affiliations.

Copyright: (C) 2022 by the authors. Licensee MDPI, Basel, Switzerland. This article is an open access article distributed under the terms and conditions of the Creative Commons Attribution (CC BY) license (https:// creativecommons.org/licenses/by/ $4.0 /)$.
1 Institute of Pharmacology and Toxicology, Technische Universität Dresden, 01307 Dresden, Germany; wener.li@tu-dresden.de (W.L.); xiaojing.luo@tu-dresden.de (X.L.); mareike.poetsch@tu-dresden.de (M.S.P.); anna.strano@tu-dresden.de (A.S.); marcel.hasse@tu-dresden.de (M.H.); robert-patrick.steiner@tu-dresden.de (R.-P.S.)

2 Institute of Clinical Pharmacology, Technische Universität Dresden, 01307 Dresden, Germany; reinhard.oertel@tu-dresden.de

3 QuoData-Quality \& Statistics GmbH, 01309 Dresden, Germany; kapil.nichani@quodata.de (K.N.); martin.schneider@quodata.de (M.S.); hettwer@quodata.de (K.H.); uhlig@quodata.de (S.U.); simon@quodata.de (K.S.)

4 Clinic for Cardiology and Pneumology, University Medical Center Göttingen, 37075 Göttingen, Germany; lukas.cyganek@med.uni-goettingen.de

5 German Center for Cardiovascular Research (DZHK), Partner Site Göttingen, 37075 Göttingen, Germany

* Correspondence: kaomei.guan@tu-dresden.de (K.G.); mario.schubert1@tu-dresden.de (M.S.)

+ These authors contributed equally to this work.

$\ddagger \quad$ These authors contributed equally to this work.

\begin{abstract}
Adverse effects of drug combinations and their underlying mechanisms are highly relevant for safety evaluation, but often not fully studied. Hydroxychloroquine (HCQ) and azithromycin (AZM) were used as a combination therapy in the treatment of COVID-19 patients at the beginning of the pandemic, leading to higher complication rates in comparison to respective monotherapies. Here, we used human-induced pluripotent stem cell-derived cardiomyocytes (iPSC-CMs) to systematically investigate the effects of $\mathrm{HCQ}, \mathrm{AZM}$, and their combination on the structure and functionality of cardiomyocytes, and to better understand the underlying mechanisms. Our results demonstrate synergistic adverse effects of AZM and HCQ on electrophysiological and contractile function of iPSC-CMs. HCQ-induced prolongation of field potential duration (FPDc) was gradually increased during 7-day treatment period and was strongly enhanced by combination with AZM, although AZM alone slightly shortened FPDc in iPSC-CMs. Combined treatment with AZM and HCQ leads to higher cardiotoxicity, more severe structural disarrangement, more pronounced contractile dysfunctions, and more elevated conduction velocity, compared to respective monotreatments. Mechanistic insights underlying the synergistic effects of AZM and HCQ on IPSC-CM functionality are provided based on increased cellular accumulation of HCQ and AZM as well as increased Cx43and Nav1.5-protein levels.
\end{abstract}

Keywords: hydroxychloroquine; azithromycin; field potential duration; conduction velocity; human induced pluripotent stem cells; cardiomyocytes; drug testing; drug interaction

\section{Introduction}

Cardiotoxicity represents one of the top reasons for drug withdrawal from clinical trials and the market, with increasing attrition rates over the past decades [1]. One of the major cardiotoxic effects is fatal cardiac arrhythmia due to direct drug interactions with cardiac electrophysiology. Therefore, preclinical testing for cardiac adverse effects 
has been focused primarily on the investigation of ion channel functions and their associated abnormalities in action potentials and conduction velocity. However, many studies reveal that mechanisms of cardiotoxicity are more complex, go beyond effects on cardiac electrophysiology [1,2], and involve dysregulation of a variety of cellular processes such as calcium cycling, cellular trafficking, mitochondrial function, and apoptosis [1]. Importantly, consequences of impaired trafficking or apoptosis may establish during prolonged exposure to a drug, especially if drug accumulation occurs even though the compound is applied in a rather low, clinically relevant concentration.

At the beginning of the worldwide pandemic caused by the severe acute respiratory syndrome coronavirus 2 (SARS-CoV-2), attempts using hydroxychloroquine (HCQ) in combination with azithromycin (AZM) reported first positive results for the treatment of SARS-CoV-2 infected (COVID-19) patients, demonstrating reinforced viral load reduction/disappearance in a small number of COVID-19 patients [3,4]. However, the follow-up clinical trials could not confirm the efficacy of treatment with HCQ or HCQ in combination with AZM [5-7]. QT interval prolongation, as reported by various groups [8-11], is considered as an adverse effect of the combined treatment with HCQ and AZM in COVID-19 patients.

Both HCQ and AZM are known to accumulate in cells, impair important cellular processes such as autophagy [12,13], and to affect cardiac electrophysiology. Conduction disorders were reported to occur in $85 \%$ of patients after chronic treatment with HCQ (or chloroquine) and represented one of the main side effects of HCQ [14,15]. Mechanistic insights from animal models revealed that acute application of HCQ reduces the heart rate by modulating the funny current $I_{f}[16,17]$. AZM, a broad-spectrum macrolide antibiotic, was considered a good safety profile until the report of a small absolute increase in cardiovascular deaths during 5 days of AZM therapy [18]. In addition, several cases of AZM-induced QT-interval prolongation were reported in the clinic $[17,19]$. So far, cardiotoxicity of HCQ and AZM was mainly studied in acute or short-term experimental settings [16,17,20-22]. There is limited knowledge about their interactions and effects on the function of human cardiomyocytes when applied in combination in a clinically relevant concentration range and duration, which may contribute to the clinically observed complications. As the mechanisms underlying HCQ- and AZM-related cardiac synergistic effects are not fully understood, the benefit-risk balance of treating COVID-19 patients with these compounds remains a dilemma for physicians.

The aim of the study was to investigate the effects of HCQ, AZM, and their combination, to identify potential synergistic drug interactions and to better understand their arrhythmia-inducing mechanisms in an in vitro human cardiomyocyte model system. Clinical practice in the treatment of COVID-19 patients and previously reported plasma concentrations of HCQ and AZM served as a rationale for the design of our study. Drug concentrations ranging from 1 to $10 \mu \mathrm{M}$ were defined based on the antiviral potency of HCQ $\left(E_{50}: 4.2 \mu \mathrm{M}\right)$ and $\mathrm{AZM}\left(\mathrm{EC}_{50}: 2.1 \mu \mathrm{M}\right)$ [23] and the reported dosages used for COVID-19 patients (600-800 mg/day for HCQ and 250-500 mg/day for AZM). The therapeutic blood levels of HCQ for systemic lupus erythematosus were $1.5 \mu \mathrm{M}$ to $6 \mu \mathrm{M}$ in patients receiving a dose of 200 or $400 \mathrm{mg}$ /day [24,25]. Although AZM plasma level was rather low, $\sim 0.3 \mu \mathrm{M}$ in patients receiving a dose of $250 \mathrm{mg}$ daily [22,26], AZM is known to accumulate rapidly in cells [22,27-31]. The use of induced pluripotent stem cell-derived cardiomyocytes (iPSC-CMs) in this study offered a robust platform to investigate the consequences of HCQ and AZM treatment on the viability, the contractile structure, contractility, and electrophysiology of human cardiomyocytes. To investigate the effects of the drugs on human cardiomyocytes reflecting different genetic backgrounds, iPSC-CMs from four different donors (1-2 iPSC lines each) without known cardiovascular disease were treated with HCQ, AZM, or their combination for 7 days (Figure S1). Afterward, they were cultured for another 7 days without the drugs (washout period). The treatment duration of 7 days was chosen based on the time course of HCQ and AZM treatment in COVID-19 patients, which varied between 5 and 10 days $[3,6,9,32]$. The duration of the washout phase was 
determined based on clinical case reports describing the reversibility of HCQ-induced QT prolongation 4-7 days after drug withdrawal $[33,34]$.

\section{Results}

\subsection{Effects of HCQ and AZM on Cell Morphology and Viability}

First, the effects of HCQ and AZM on the morphology of iPSC-CMs were investigated. Treatments with AZM and HCQ, in particular at higher concentrations, caused the formation of vacuole-like structures within the cells (Figures 1A and S2A), which persisted until 7 days after drug washout (Figures 1D and S2B). Overview images (Figures S1A and S2A) and cell nucleus counting (Figure S2C) showed reduction in total cell number after 7 days of the combined treatment with $10 \mu \mathrm{M}$ HCQ and $10 \mu \mathrm{M}$ AZM. Importantly, treatment with vehicle had no influence on viability or cell number (Figure S3A-C). Cells treated with $10 \mu \mathrm{M} H C Q$ alone or in combination with $1 \mu \mathrm{M}$ AZM showed a progressive cell death (Figure 1B). The MTT assay revealed that 7-day combination treatment with $10 \mu \mathrm{M}$ HCQ and $10 \mu \mathrm{M}$ AZM led to less than $50 \%$ of cells at a viable and metabolically active state (Figure 1B), whereas HCQ $(1 \mu \mathrm{M}$ or $3 \mu \mathrm{M})$ alone or in combination with AZM $(1 \mu \mathrm{M}$ or $10 \mu \mathrm{M}$ ), respectively, did not significantly affect metabolic activity of iPSC-CMs (Figure 1B). Significantly higher rates of cell death were also observed based on increased lactate dehydrogenase (LDH) activity in the cell supernatant of the groups treated with $10 \mu \mathrm{M}$ HCQ alone and in combination with AZM $(1 \mu \mathrm{M}$ or $10 \mu \mathrm{M})$ (Figure 1C). In contrast to HCQ, $10 \mu \mathrm{M}$ AZM alone showed no effect on cell viability using both MTT and LDH activity assays (Figure 1B,C).

After 7-day drug washout, AZM-induced accumulation of vacuole-like structures was reduced, consistent with a previously published study [13]. However, the cytotoxic effect of $10 \mu \mathrm{M}$ HCQ became more evident. The MTT assay revealed a further decrease in viability of cells treated with $10 \mu \mathrm{M}$ HCQ alone or in combination with $1 \mu \mathrm{M} \mathrm{AZM}$, which was consistent with reduced cell confluence (Figure 1D,E). Notably, due to significantly reduced cell numbers in groups treated with $10 \mu \mathrm{M}$ HCQ alone or in combination with AZM as well as the daily medium change, $\mathrm{LDH}$ activity in the supernatant is not representative in these samples after the drug washout (Figure 1F). Treatment of cells with lower drug concentrations ( 1 or $3 \mu \mathrm{M}$ HCQ, 1 or $10 \mu \mathrm{M}$ AZM) did not affect cell viability (Figure 1E,F). These results demonstrate the toxicity of HCQ at high concentrations, which is further increased in the presence of AZM.

\subsection{HCQ and AZM Affect the Structural Organization of iPSC-CMs}

To investigate the effects of HCQ and AZM on PPSC-CM area, sarcomere organization, and sarcomere length, we performed immunofluorescence staining to detect $\alpha$-actinin. To evaluate the effect of AZM and HCQ on cell area, iPSC-CMs were seeded at low density to monitor single cells. Single cells were less resistant to drug treatment compared to cells in monolayer by showing severe morphological changes and cell death, in particular, under treatment with $10 \mu \mathrm{M}$ HCQ and $10 \mu \mathrm{M}$ AZM either alone or in combination (Figure 2A). Therefore, structural analyses of iPSC-CMs were only performed for treatments with lower drug concentrations, for which cell detachment was less evident.

The 7-day treatment with $1 \mu \mathrm{M}$ AZM alone resulted in a slight increase in cell area (Figure 2B), but this did not persist, and rather resulted in a slight decrease after drug washout (Figure 2C). After 7-day treatment with $3 \mu \mathrm{M}$ HCQ alone, iPSC-CMs showed a reduction in cell area, which was not obvious in the groups treated with $1 \mu \mathrm{M} \mathrm{HCQ}$ alone or with HCQ $(1$ and $3 \mu \mathrm{M})$ in combination with $1 \mu \mathrm{M}$ AZM (Figure 2A,B). However, after the drug washout, iPSC-CMs treated with 1 and $3 \mu \mathrm{M}$ HCQ alone or in combination with $1 \mu \mathrm{M}$ AZM showed smaller cell areas compared to control cells, indicating persistent cellular shrinking (Figure 2C). 
A

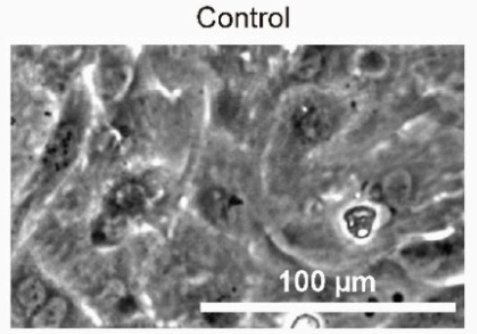

$1 \mu \mathrm{M} \mathrm{AZM}$

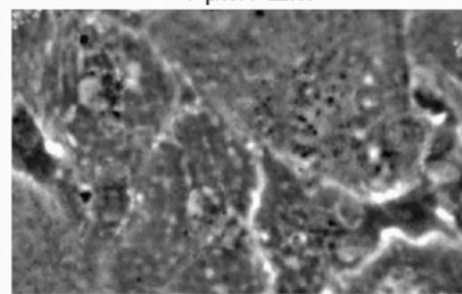

B

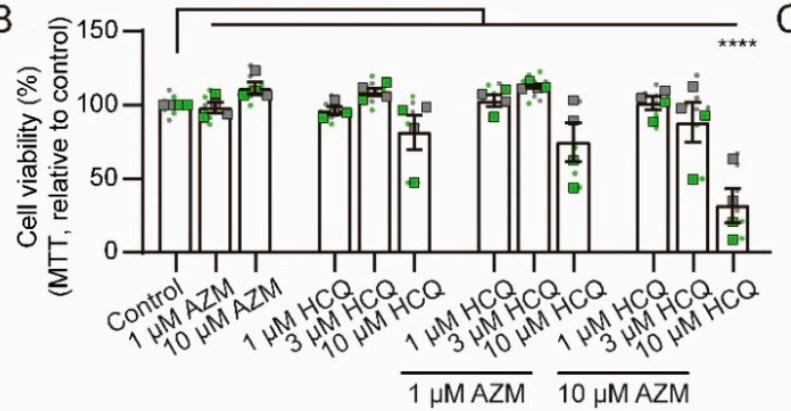

D

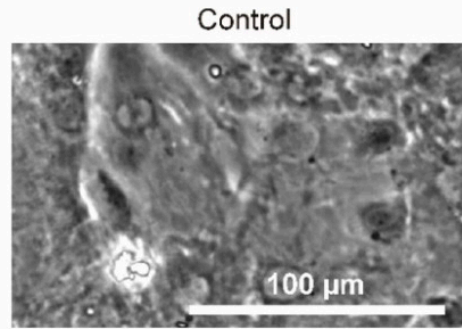

$1 \mu \mathrm{M}$ AZM

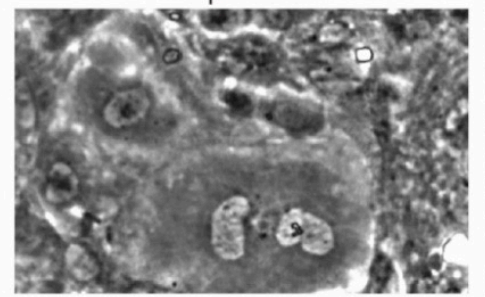

E

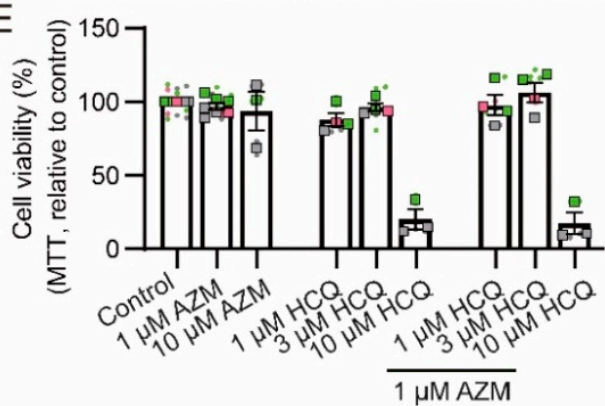

$1 \mu \mathrm{M} \mathrm{HCQ}$

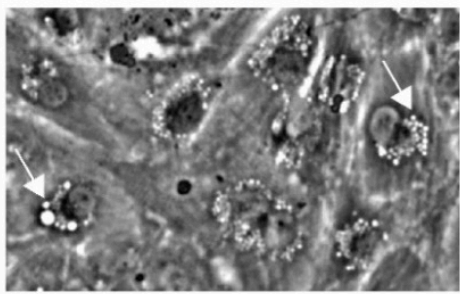

$10 \mu M$ AZM

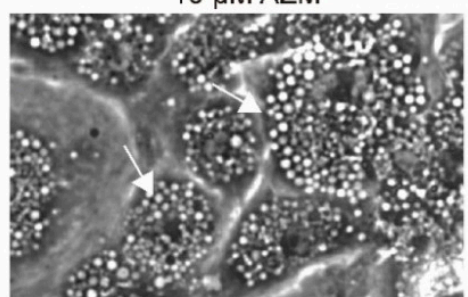

$10 \mu \mathrm{M} \mathrm{HCQ}$

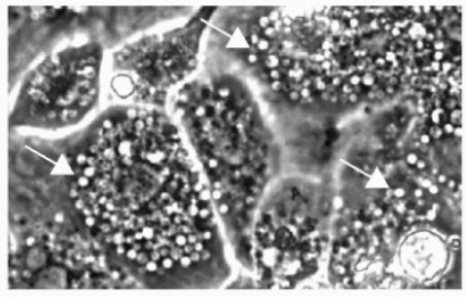

$1 \mu \mathrm{M} \mathrm{AZM}+10 \mu \mathrm{M} \mathrm{HCQ}$

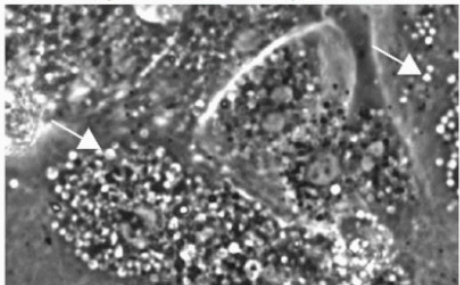

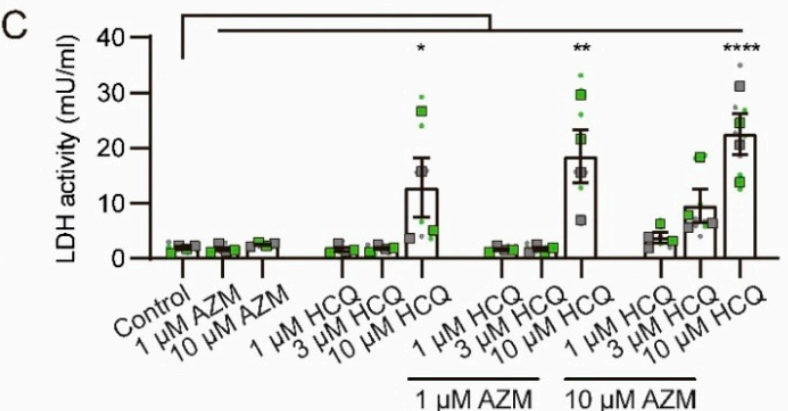

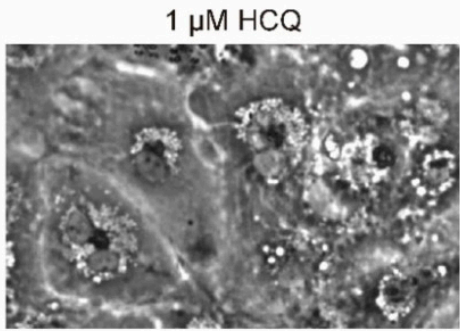

$10 \mu \mathrm{M} \mathrm{HCQ}$

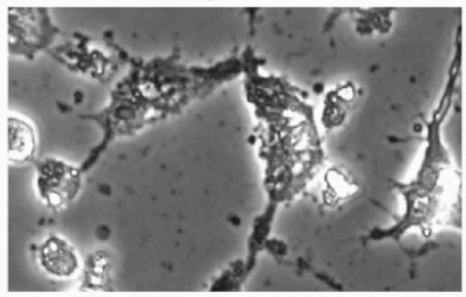

$1 \mu \mathrm{M} \mathrm{AZM}+10 \mu \mathrm{M} \mathrm{HCQ}$
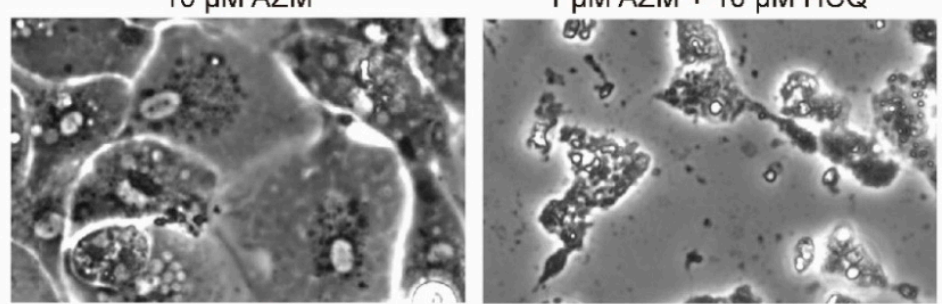

F

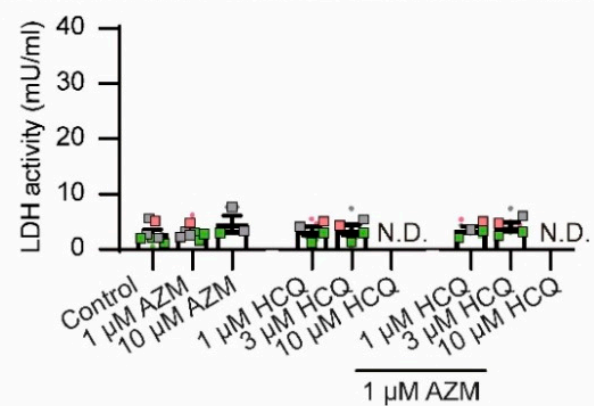

Figure 1. Morphological changes and cytotoxicity in iPSC-CMs treated with HCQ and AZM. (A) Representative brightfield images depicting morphology of iPSC-CMs after 7-day treatments with HCQ and AZM in different concentrations. Arrows indicate formation of vacuoles. Scale bar: $100 \mu \mathrm{m}$. 
(B) Cell viability after 7-day drug treatment as determined by measurement of formazan formation in the MTT assay. (C) LDH activity detected in cell supernatants after 7-day drug treatment. (D) Representative brightfield images depicting morphology of iPSC-CMs after 7-day drug treatment and 7-day washout period. Even after washout, iPSC-CMs treated with a combination of high concentrations of AZM and HCQ show severe morphological changes and increased cell death. Scale bar: $100 \mu \mathrm{m}$. (E) Cell viability after 7-day drug washout as determined by using the MTT assay. (F) LDH activity detected in supernatants after 7-day drug washout. Data represent technical replicates (points) and means (squares) of each experiment, $N=3-7$ independent experiments using iPSC-CMs from 3 healthy donors (iBM76.1, iBM76.3 in green; iWTD2.1, iWTD2.3 in grey, isWT7.22 in pink). Lines and errors show overall mean and SEM. Statistical analysis was performed using one-way ANOVA and Tukey's multiple comparison test. ${ }^{*} p<0.05,{ }^{* *} p<0.01,{ }^{* * *} p<0.0001$. N.D.- - not determined.

To quantify the effect of HCQ and AZM on sarcomeric organization in iPSC-CMs, the proportion of cells with structurally organized and disorganized sarcomeres were manually determined based on the images of iPSC-CMs stained for $\alpha$-actinin. Cells with evenly distributed intact sarcomeres across the cell body (occupying $>80 \%$ of the cell area) were classified as structurally organized (Figure 2D, left), while cells with intact sarcomeres distributed exclusively in the center or periphery or cells lacking clearly organized ladder-like sarcomeres were classified as structurally disorganized (Figure 2D, right). Under basal conditions, $61 \pm 6 \%$ of iPSC-CMs were classified as structurally organized (Figure 2E). The relatively high portion of cells with disorganized sarcomeres at basal condition might result from the immaturity of iPSC-CMs undergoing sarcomere assembly. Treatment with $1 \mu \mathrm{M}$ AZM and $1 \mu \mathrm{M}$ HCQ alone revealed no effect on the sarcomere organization of iPSC-CMs. An increase in the percentage of structurally disorganized cells was found in cells treated with $3 \mu \mathrm{M}$ HCQ alone $(p=0.055)$ or in combination with $1 \mu \mathrm{M}$ AZM ( $p<0.0001$, Figure 2E).

As another important aspect of iPSC-CM structure, the sarcomere length was measured in the population of structurally organized cells (Figure 2D, left). The sarcomere length of iPSC-CMs at basal condition was determined as $2.04 \pm 0.05 \mu \mathrm{m}$, which is comparable to a sarcomere length of $\sim 2.2 \mu \mathrm{m}$ observed in mature cardiomyocytes [35]. After a 7-day treatment with $1 \mu \mathrm{M}$ AZM, $3 \mu \mathrm{M}$ HCQ, or the combination of HCQ (1 and $3 \mu \mathrm{M})$ and $1 \mu \mathrm{M}$ AZM, iPSC-CMs showed a significant reduction in sarcomere length, which was not obvious in the group treated with $1 \mu \mathrm{M}$ HCQ alone (Figure $2 \mathrm{~F}$ ). The strongest reduction in sarcomere length was observed in the group treated with $1 \mu \mathrm{M}$ AZM combined with $3 \mu \mathrm{M}$ HCQ. After the subsequent washout period for 7 days, sarcomere length remained strongly reduced in groups treated with $3 \mu \mathrm{M} H C Q$ alone and HCQ in combination with AZM and slightly reduced in iPSC-CMs treated with $1 \mu \mathrm{M} \mathrm{AZM}$ or $1 \mu \mathrm{M}$ HCQ alone (Figure 2G).

Taken together, these results highlight the negative effect of HCQ and AZM treatments on the structural characteristics of iPSC-CMs and the persistence of their adverse effects even after drug washout for 7 days. 
A
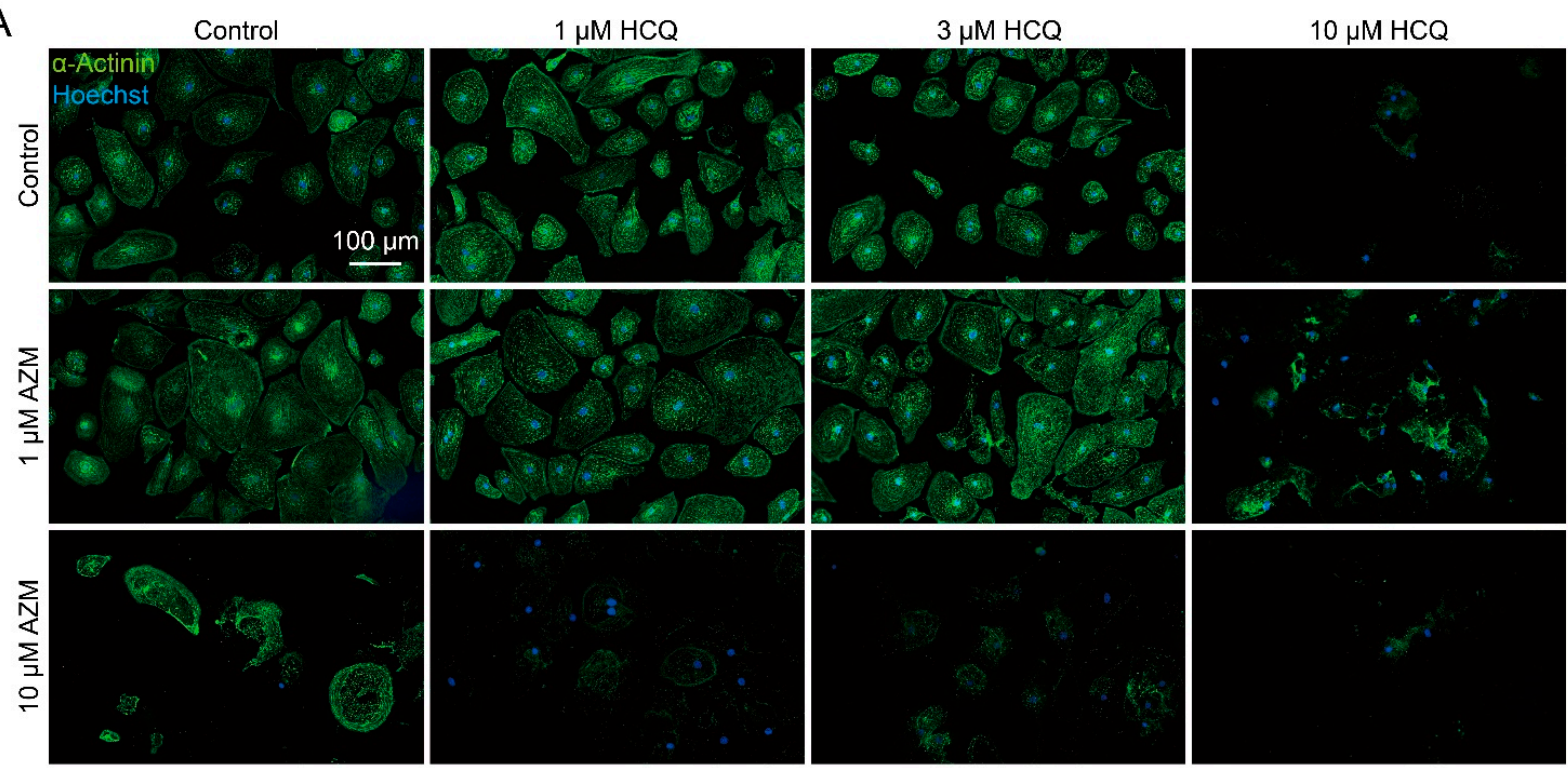

B
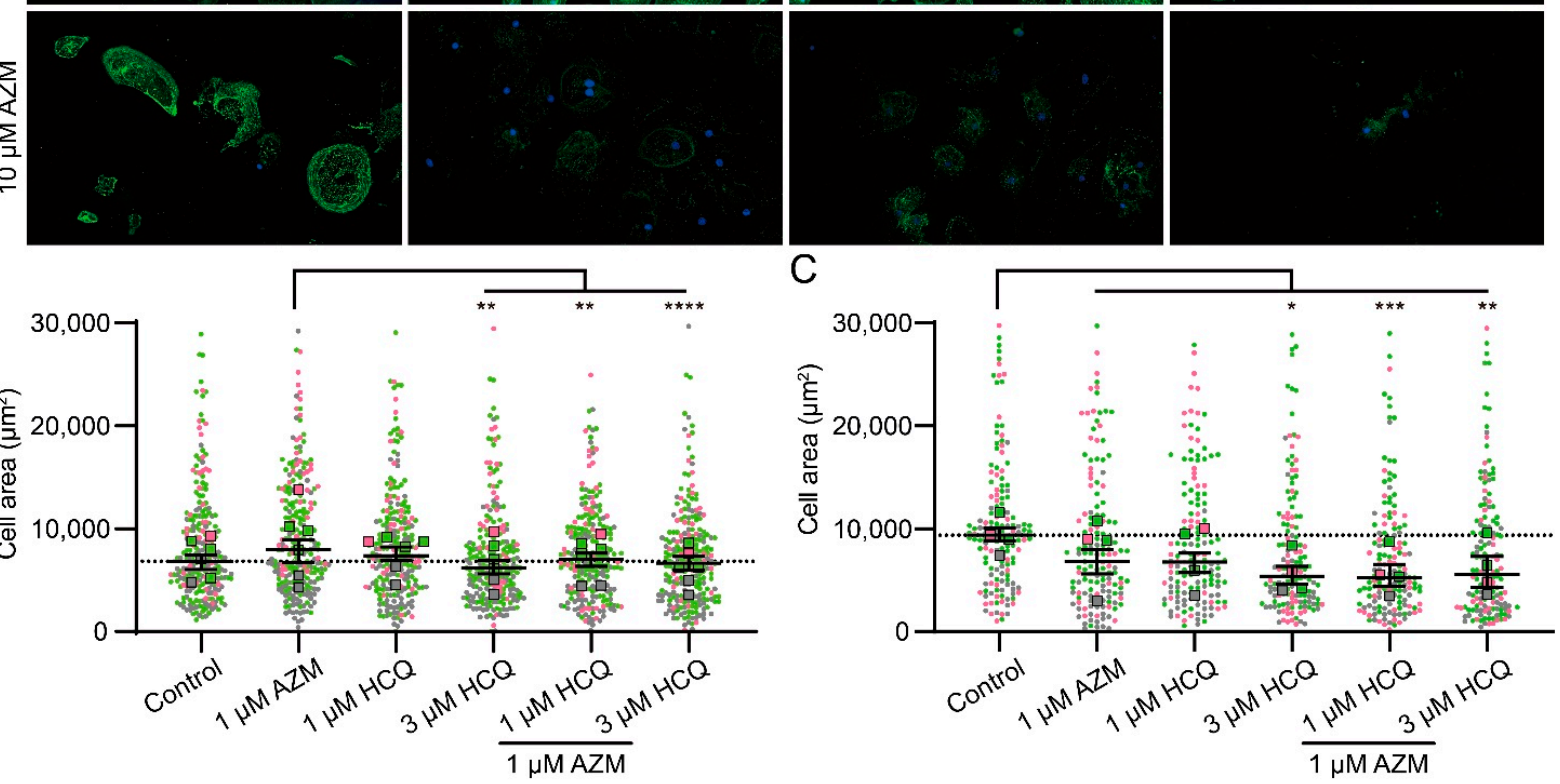

$\mathrm{D}$

$E$

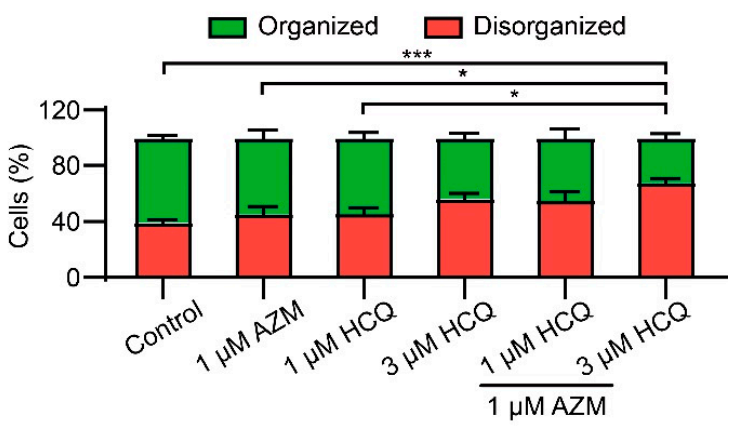

$\mathrm{F}$

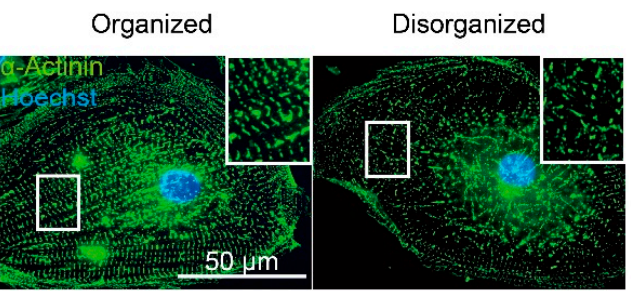

G
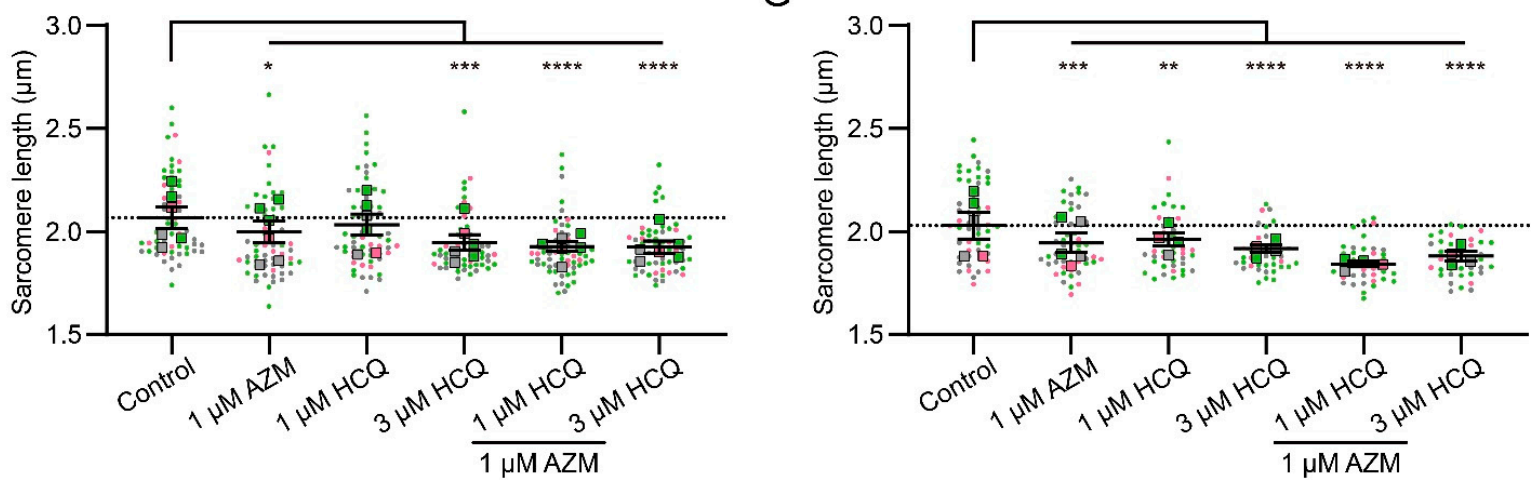

Figure 2. HCQ and AZM cause sarcomeric disorganization in iPSC-CMs. (A) Representative images of $\alpha$-actinin immunostained iPSC-CMs treated with different concentrations of HCQ and AZM for 
7 days. (B,C) Analysis of cell areas after 7-day drug treatment (B) and after subsequent 7-day washout (C). A total of $n=160-240$ cells (40 per experiment) from 6 (B) or 4 (C) independent experiments per condition were analyzed. Points represent values of single cells and squares the median values of individual experiments. Lines indicate median and $95 \% \mathrm{CI}$ of the overall population. (D) Representative images of structurally organized and disorganized iPSC-CMs after drug treatment for 7 days. (E) Percentage of structurally organized and disorganized iPSC-CMs after 7-day drug treatment. Mean and SEM of 5 independent experiments $(n=96-272$ cells analyzed per condition from each experiment, same number of cells at different conditions analyzed within one experiment) are shown. (F,G) Sarcomere length after 7-day drug treatment (F) and after 7-day washout (G). Mean and SEM of $n=50-60$ cells (10 per experiment) from $6(\mathbf{F})$ or $5(\mathbf{G})$ independent experiments are shown. Data plots in (F), and (G) show technical replicates (dots) and mean values (squares). Colors indicate iPSC-CM differentiations from 3 healthy donors (iBM76.1, iBM76.3 in green; iWTD2.1, iWTD2.3 in grey, isWT7.22 in pink). Statistical analysis was performed using one-way ANOVA and Tukey's multiple comparison test. ${ }^{*} p<0.05,{ }^{* *} p<0.01,{ }^{* * *} p<0.001,{ }^{* * * *} p<0.0001$.

\subsection{HCQ and AZM Alter the Contractility of iPSC-CMs}

The effects of HCQ and AZM on the beating property of iPSC-CMs were investigated using video-based motion vector analysis (Figure S4A). This method allows the quantification of specific parameters of contraction and relaxation [36,37]. As a quality control, all beating parameters remained unchanged for cells cultured with $0.1 \%$ DMSO (vehicle) during the 7-day treatment (Figure S3). A progressive alteration of cardiomyocyte beating properties was observed in the presence of AZM and HCQ during the 7-day treatment period (Figures 3 and S4B-G, Videos S1 and S2). While AZM $(1 \mu \mathrm{M})$ and HCQ $(1 \mu \mathrm{M}$ and $3 \mu \mathrm{M})$ alone had no or less effect on the beating parameters of iPSC-CMs during the 7-day treatment (Figure 3B-E), strong changes were observed in iPSC-CMs treated with AZM $(10 \mu \mathrm{M})$ and HCQ $(10 \mu \mathrm{M})$ alone or with AZM $(1$ and $10 \mu \mathrm{M})$ in combination with HCQ $(1,3$, and $10 \mu \mathrm{M})$ (Figures 3B-E and S4B-G). Notably, treatment with $10 \mu \mathrm{M}$ HCQ alone or combined with $1 \mu \mathrm{M}$ or $10 \mu \mathrm{M}$ AZM led to stopping of beating or strongly distorted motion in some cultures of iPSC-CMs, which could not be included in the analysis (Figure 3A,B). With respect to beating rate (Figure 3B and S4B), iPSC-CMs treated with $10 \mu \mathrm{M}$ AZM alone showed an increase in beating rate at day 1 but a decrease at day 7 (Figures 3B and S4B), while $10 \mu \mathrm{M}$ HCQ alone progressively increased the beating rate of iPSC-CMs from day 1 onwards (Figure 3B and S4B). A combination of AZM (1 or $10 \mu \mathrm{M})$ with $10 \mu \mathrm{M}$ HCQ led to even higher beating rates than $10 \mu \mathrm{M}$ HCQ alone (Figure 3B). Moreover, an increased beating rate was observed in the group treated with $3 \mu \mathrm{M}$ HCQ in combination with $10 \mu \mathrm{M}$ AZM, which was absent in the cells treated with $3 \mu \mathrm{M} \mathrm{HCQ}$ alone (Figure $3 \mathrm{~B}$ ), indicating the synergistic effects of both drugs. In terms of contraction time and relaxation time, $10 \mu \mathrm{M}$ AZM alone showed a progressive reduction, similar to the group treated with $10 \mu \mathrm{M} \mathrm{HCQ}$ alone during the 7-day treatment (Figures 3C,D and S4D,F). The combination of HCQ and AZM enhanced the decrease of contraction and relaxation time in a concentration- and time-dependent manner (Figure 3C,D).

Overall, these data demonstrate that AZM and HCQ directly affect the beating rate, as well as the contraction and relaxation behavior of iPSC-CMs in a concentration- and time-dependent manner, while the combination of AZM and HCQ enhances the effects of $\mathrm{HCQ}$ on increasing the beating rate as well as on decreasing contraction time and relaxation time of iPSC-CMs. 
Day 3

Day 7

A

$\rightarrow$ Control $\quad \longrightarrow 10 \mu \mathrm{M} \mathrm{AZM} \quad \longleftarrow 10 \mu \mathrm{M} \mathrm{HCQ} \quad \longleftarrow 10 \mu \mathrm{M} \mathrm{AZM}+3 \mu \mathrm{M} \mathrm{HCQ}$
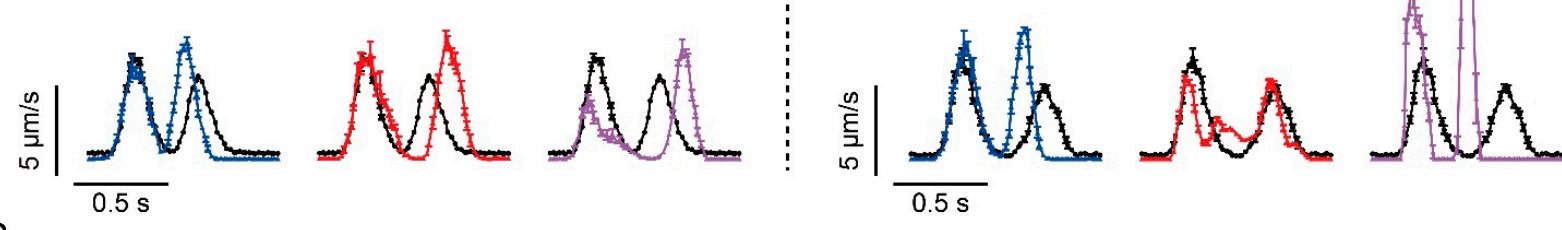

B
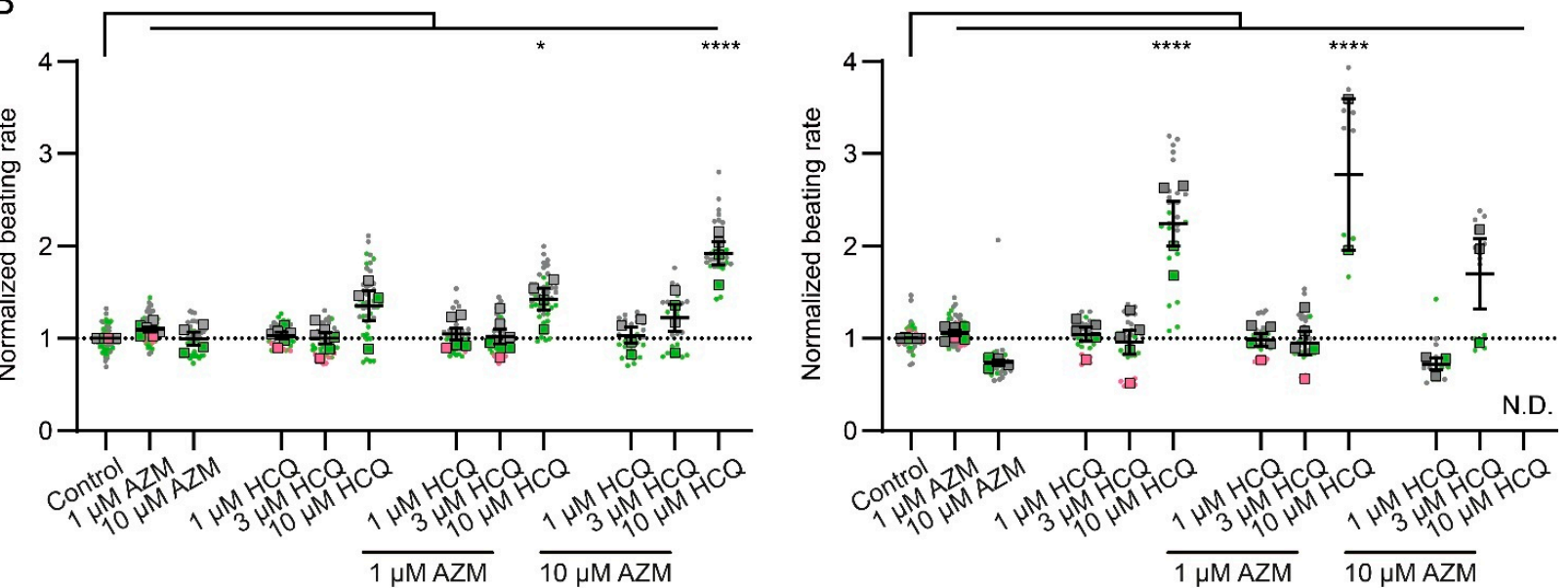

C

$\overline{1 \mu \mathrm{M} \mathrm{AZM}} \quad \overline{10 \mu \mathrm{M} \mathrm{AZM}}$

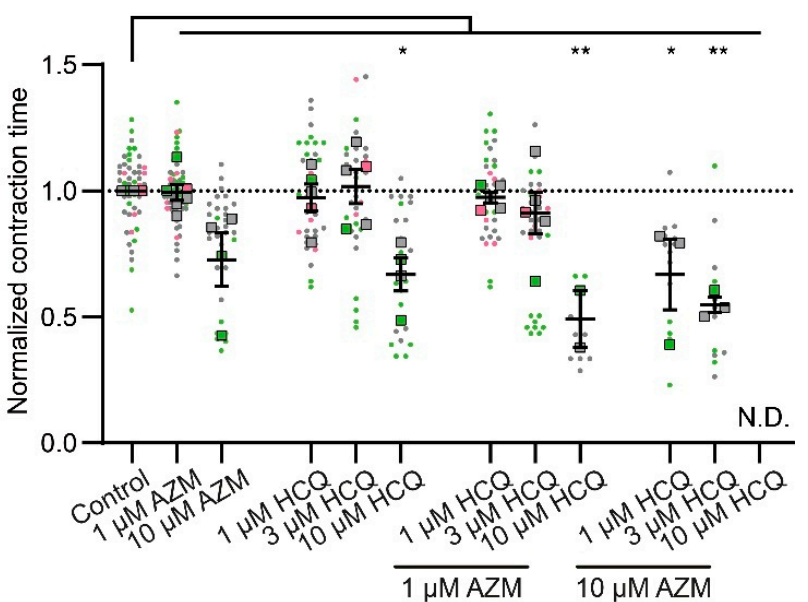

$\mathrm{D}$
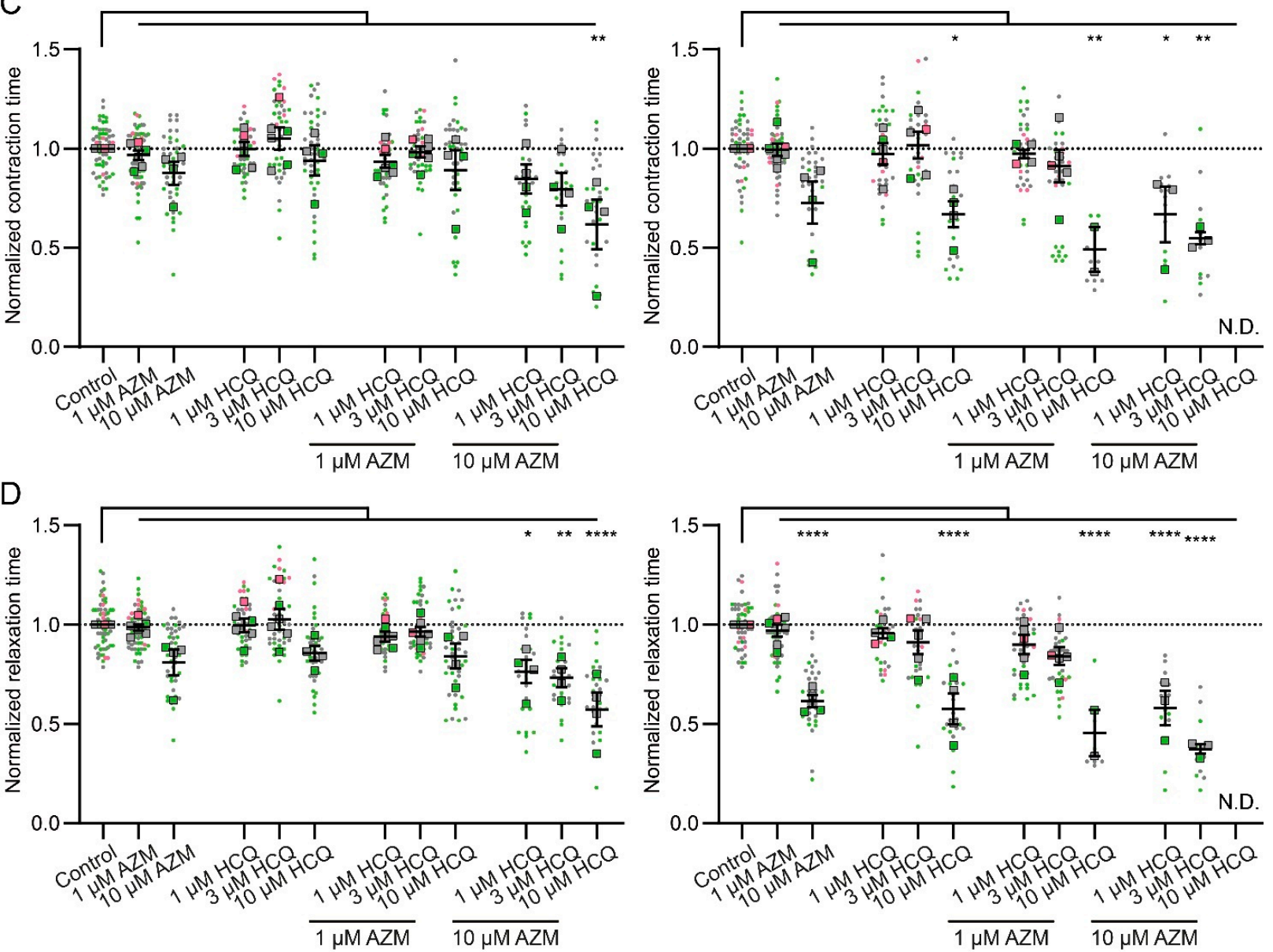

Figure 3. Contractile dysfunctions in iPSC-CMs treated with HCQ and AZM. (A) Representative motion traces observed in iPSC-CMs using vector-based quantification on treatment day 3 (left) and 
7 (right). Values represent mean and SEM of motions from aligned contraction-relaxation cycles of a representative video. (B-D) Effects of AZM and HCQ alone as well as their combination on the beating rate (B), contraction time (C), and relaxation time (D) on treatment day 3 (left) and 7 (right). Data represent technical replicates (points, $n=9-54$ videos per condition) and means (squares) of each experiment, $N=4-6$ independent experiments using iPSC-CMs from 3 healthy donors (iBM76.1, iBM76.3 in green; iWTD2.1, iWTD2.3 in grey, isWT7.22 in pink). Due to the toxic effects of HCQ or AZM at higher concentrations or in combination, fewer videos could be analyzed under these conditions. Lines show overall mean values and SEM. Statistical analysis based on the mean values of the individual experiments using one-way ANOVA and Tukey's multiple comparison test. ${ }^{*} p<0.05$, ** $p<0.01,{ }^{* * * *} p<0.0001$.

\subsection{HCQ and AZM Lead to the Prolongation of Field Potential Duration in iPSC-CMs}

To assess the effect of HCQ and AZM on the heart rhythm, the field potential (FP) analysis in iPSC-CMs were performed using the multi-electrode array (MEA) technique. As shown in Figure 4A,B, the corrected FP duration (FPDc) in the control group remained stable while $1 \mu \mathrm{M}$ AZM showed no effect on the FPDc during the 14-day recording (7-day drug treatment and subsequent 7-day washout). However, $10 \mu \mathrm{M}$ AZM slightly shortened the FPDc of iPSC-CMs, and drug washout could not restore it to the basal level (Figure 4A,B). Treatment with HCQ at low concentrations (1 $\mu \mathrm{M}$ and $3 \mu \mathrm{M})$ had no effect on FPDc, however, iPSC-CMs treated with $10 \mu \mathrm{M}$ HCQ showed a prolonged FPDc from day 2, which kept rising until day 7 (Figure 4A,C). The prolongation of FPDc induced by $10 \mu \mathrm{M} \mathrm{HCQ}$ was reversible, as drug washout gradually eliminated this effect.

When $1 \mu \mathrm{M}$ AZM was combined with HCQ $(1,3$ or $10 \mu \mathrm{M})$, similar effects as HCQ alone were observed, showing the prolongation of FPDc only with $10 \mu \mathrm{M} H C Q$, but to a lesser extent (Figure 4D). Notably, the combination of $10 \mu \mathrm{M}$ AZM with $3 \mu \mathrm{M} \mathrm{HCQ}$ significantly and reversibly prolonged the FPDc of iPSC-CMs, which was not observed in cells treated with the combination of $10 \mu \mathrm{M}$ AZM and $1 \mu \mathrm{M}$ HCQ (Figure 4). When we combined $10 \mu \mathrm{M}$ AZM with $10 \mu \mathrm{M}$ HCQ, the prolonged FPDc in iPSC-CMs was evident from day 3 to day 8 (Figure $4 \mathrm{E}$ ). However, we observed that 55\% of iPSC-CMs failed to reveal FP and showed cell death on day 8 (the first day of washout), and $82 \%$ of cultures stopped beating at the end of the experiment (Figures 4A,E and S5, Table S1). In terms of beating frequency, $10 \mu \mathrm{M}$ AZM caused a significant increase in spontaneous beating frequency on day 1 but a lower beating frequency from day 4 onwards (Figure S6A) whereas $10 \mu \mathrm{M}$ HCQ led to a significant increase from day 1 onwards (Figure S6B), which is in line with the results observed in the contractility experiments. Interestingly, most drug-treated iPSC-CMs had a slower beating rate than the control group during the washout period (Figure S6).

\subsection{HCQ and AZM Independently and Synergistically Augment the Conduction Velocity of iPSC-CMs}

Since conduction disorders were the most frequent side effect that appeared in COVID-19 patients who were administrated with HCQ and AZM [38], we examined the impact of the two drugs on cardiac conduction velocity (CV) in iPSC-CM model. As shown in Figure 5, CV of iPSC-CMs in the control group remained stable during the two-week experiment. While cells treated with $1 \mu \mathrm{M}$ AZM showed a similar conduction trajectory and $\mathrm{CV}$ as in the control group, $10 \mu \mathrm{M}$ AZM led to changes in trajectory and significantly augmented CV in iPSC-CMs, starting on day 3 after drug treatment, but reversing on day 3 after drug washout (Figure 5A,B). Similar to AZM, HCQ also resulted in changes in conduction trajectory and increases in the CV of iPSC-CMs in a concentration-dependent pattern (Figure 5A,C). The addition of $1 \mu \mathrm{M}$ AZM enhanced the effects caused by HCQ alone (Figure 5D). Furthermore, when $10 \mu \mathrm{M}$ AZM was applied in addition to HCQ (1, 3, and $10 \mu \mathrm{M})$, iPSC-CMs from all three groups showed significantly faster transmission of electrical signals (Figure 5A,E). 
A

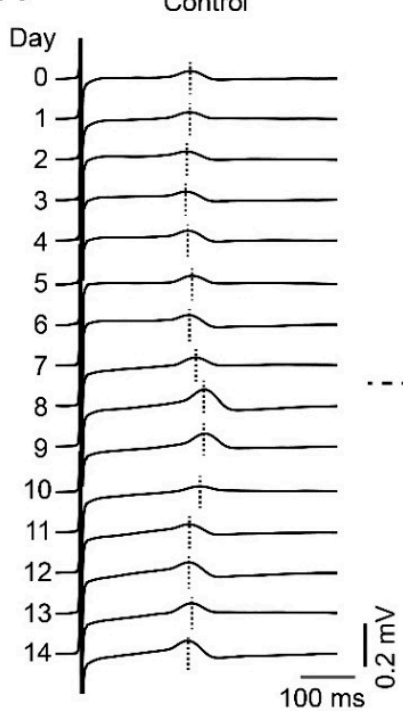

B

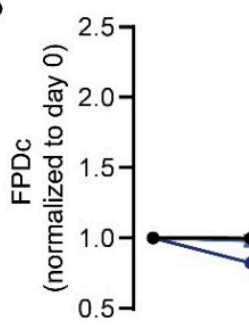

$10 \mu \mathrm{M}$ AZM

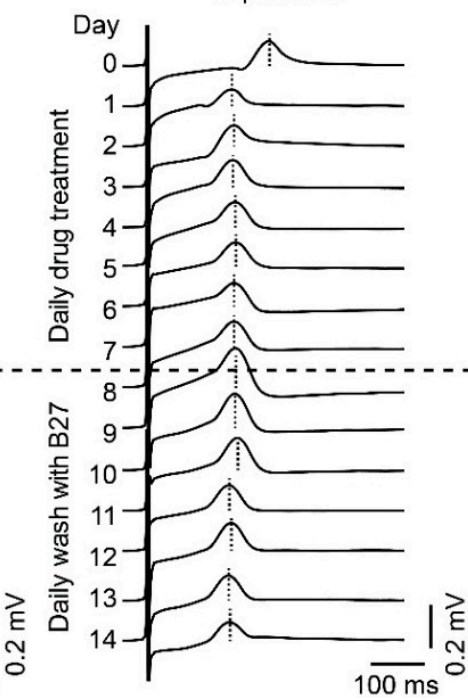

Daily drug treatment

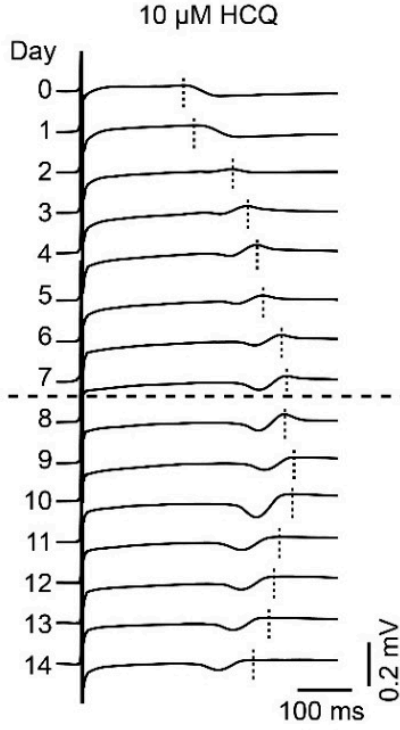

$10 \mu \mathrm{M} A Z M+10 \mu \mathrm{M}$

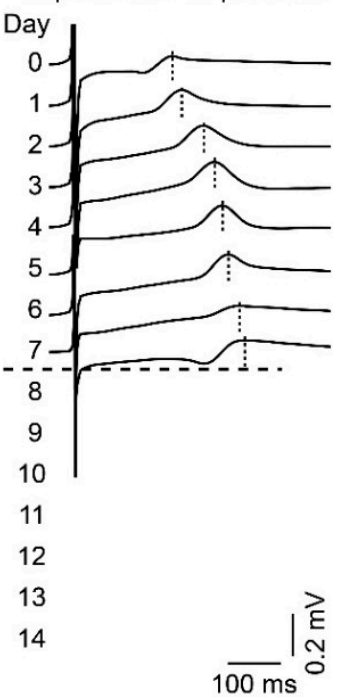

C

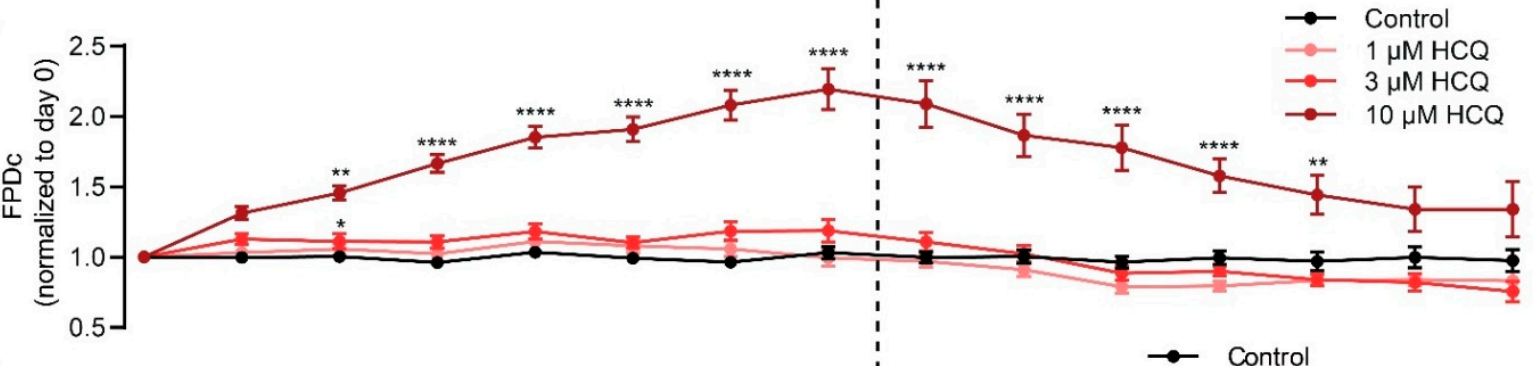

D

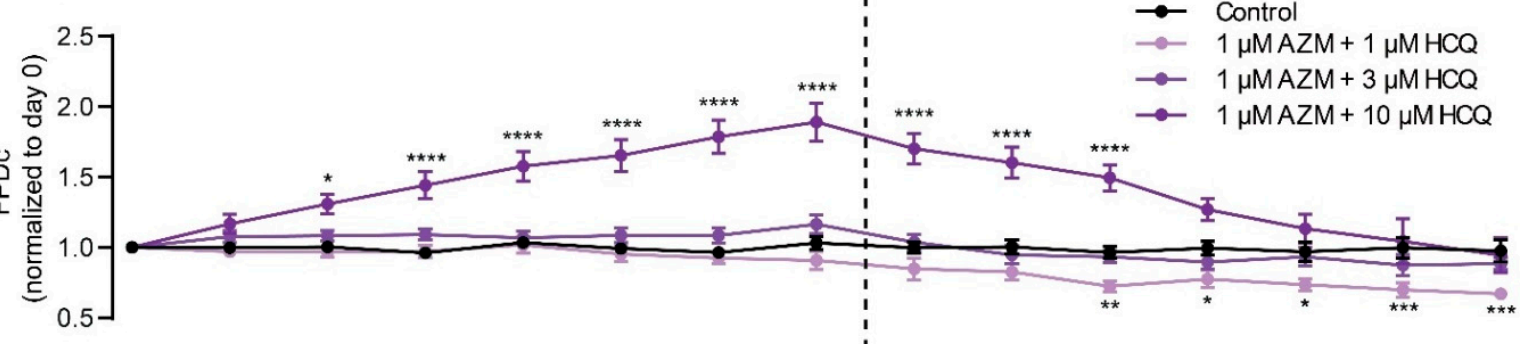

E

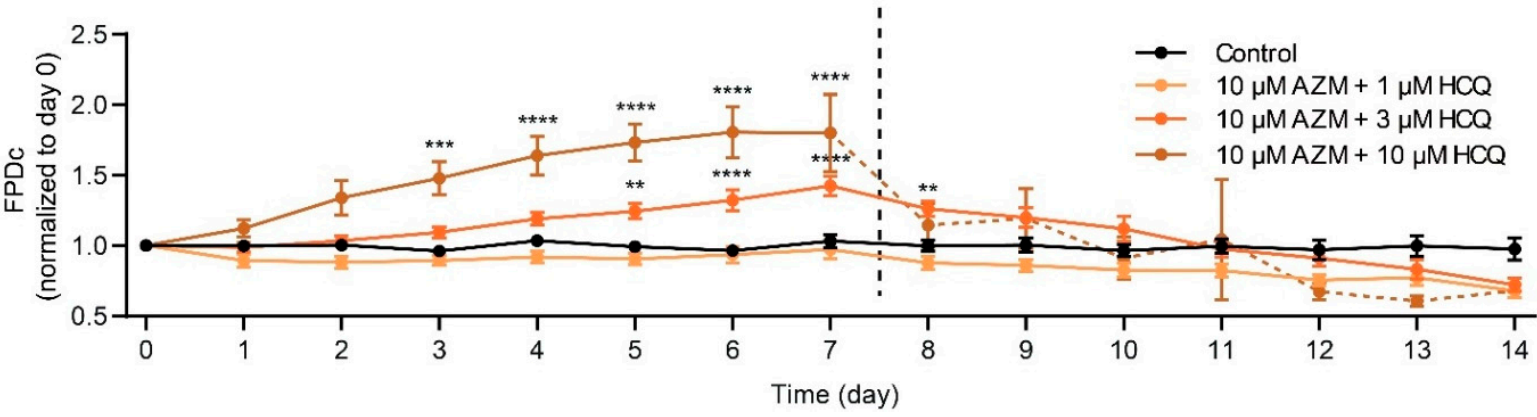

Figure 4. Effects of HCQ and AZM on the field potential duration of iPSC-CMs. (A) Representative recordings of extracellular FP in spontaneous beating iPSC-CMs under different treatment conditions. 
iPSC-CMs treated with $10 \mu \mathrm{M}$ AZM and $10 \mu \mathrm{M}$ HCQ in combination stopped beating at day 8 (one day after initiation of washout). (B,C) Effect of AZM (B) or HCQ (C) on the corrected FPD (FPDc, normalized to day 0) during 7-day treatment and subsequent 7-day washout. (D,E) Effects of HCQ $(1,3$, and $10 \mu \mathrm{M})$ combined with $1 \mu \mathrm{M}$ AZM (D) or $10 \mu \mathrm{M}$ AZM (E) on FPDc during 7-day treatment and following 7-day washout. iPSC-CMs derived from four donors were used for MEA recording. For the initial recording (day 0), $10 \leq n \leq 13$ for all conditions. Spontaneous beating states of iPSCCMs are listed in Table S1. Two-way ANOVA with Bonferroni post-hoc test was used for statistical evaluation ${ }^{*} p<0.05,{ }^{* *} p<0.01,{ }^{* * *} p<0.001$, and $\left.{ }^{* * *} p<0.0001\right)$.

\subsection{HCQ and AZM Synergistically Enhance the Expression of Cx43 and Alter the Steady-State} Kinetics of $\mathrm{I}_{\mathrm{Na}}$ in iPSC-CMs

To gain insights into the molecular mechanism of HCQ/AZM-induced CV augmentation, we analyzed expression of Nav1.5 and Cx43, which are crucial to maintaining electrical signal propagation between CMs [39]. Compared to the control group, the expression of Nav1.5 was slightly, but not significantly, higher in iPSC-CMs treated with $10 \mu \mathrm{M}$ HCQ for 7 days $(p>0.05$, Figure 6A,B). Treatment with $10 \mu \mathrm{M}$ AZM did not change Nav1.5 protein levels $(p>0.05$, Figure 6A,B). Importantly, when we applied $10 \mu \mathrm{M}$ HCQ combined with $10 \mu \mathrm{M}$ AZM to iPSC-CMs, we observed a two-fold increase in Nav1.5 protein expression $(p=0.08$, Figure $6 \mathrm{~A}, \mathrm{~B})$. In terms of Cx43, 7-day treatment with $10 \mu \mathrm{M}$ HCQ significantly increased the protein expression by three-fold $(p<0.01$, Figure $6 \mathrm{~A}, \mathrm{C})$. While treatment with $10 \mu \mathrm{M}$ AZM alone only slightly increased the Cx43 expression $(p>0.05)$, the combination of $10 \mu \mathrm{M}$ HCQ and $10 \mu \mathrm{M}$ AZM synergistically quadrupled the expression of Cx43 compared to the control group (Figure 6A,C). Similar results were observed using immunofluorescence staining, revealing a higher expression as well as a strong intracellular accumulation of Cx43 in iPSC-CMs treated with $10 \mu \mathrm{M} H C Q, 10 \mu \mathrm{M}$ AZM, and their combination (Figure 6D).

To further investigate the impact of HCQ and AZM on the function of cardiac sodium channel, we recorded $I_{N a}$ in cells treated with $10 \mu \mathrm{M}$ HCQ and/or $10 \mu \mathrm{M}$ AZM for 7 days using an automated patch-clamp technique (Figure S7). Compared to the control group, iPSC-CMs treated with $10 \mu \mathrm{M}$ AZM alone showed increased membrane capacitances (an indicator for cell size), while cells treated with $10 \mu \mathrm{M}$ HCQ in combination with $10 \mu \mathrm{M}$ AZM showed lower membrane capacitances (Figure S7B). We could not observe differences regarding the current density of $I_{\mathrm{Na}}$ in the four groups, except that the reversed current at $+70 \mathrm{mV}$ was smaller in cells treated with combined HCQ and AZM (Figure S7A,C). However, both drugs markedly modified the gating properties of cardiac sodium channel. Compared to the control group, the steady-state activation curves were leftwards shifted in the groups treated with HCQ alone or in combination with AZM, but not in iPSC-CMs treated with AZM alone (Figure S7D). Moreover, the steady-state inactivation curves of all the three groups treated with the drugs showed a rightwards shift (Figure S7E).

\subsection{HCQ and AZM Accumulate in iPSC-CMs}

As HCQ and AZM have been reported to accumulate in lysosomes and endosomes, we analyzed the levels of HCQ and AZM in lysates of iPSC-CMs after a 7-day drug treatment using mass spectrometry. Due to reduced cell viability in combination treatments with higher concentrations of HCQ and AZM, we did not include these groups in the analysis. Our data reveal that cellular levels of HCQ (Figure 7A) and AZM (Figure 7B) increased in a concentration-dependent manner after the 7-day treatment. Interestingly, levels of HCQ in iPSC-CMs treated with $1 \mu \mathrm{M}$ HCQ combined with $10 \mu \mathrm{M}$ AZM were much higher than those in cells treated with $1 \mu \mathrm{M}$ HCQ alone (Figure 7A) and accumulation of AZM was increased by co-treatment with $1 \mu \mathrm{M}(p=0.06)$ or $3 \mu \mathrm{M}(p=0.12) \mathrm{HCQ}$ (Figure 7B). These data indicate that the combined treatments with HCQ and AZM facilitate cellular accumulation of HCQ and AZM and provide further evidence for the synergistic effects of AZM and HCQ through increased cellular accumulation. 
A

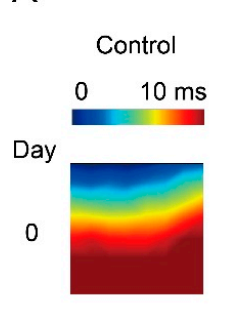

1

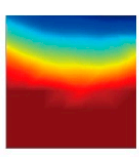

3

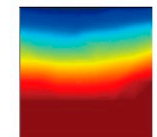

5
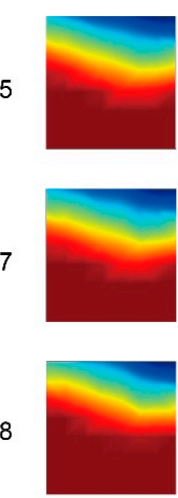

10

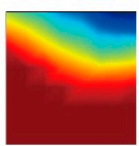

12

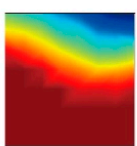

14

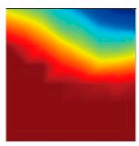

$10 \mu \mathrm{M}$ AZM

$10 \mu \mathrm{M}$ AZM $10 \mu \mathrm{M} \mathrm{HCQ} \quad 10 \mu \mathrm{M} \mathrm{HCQ}$
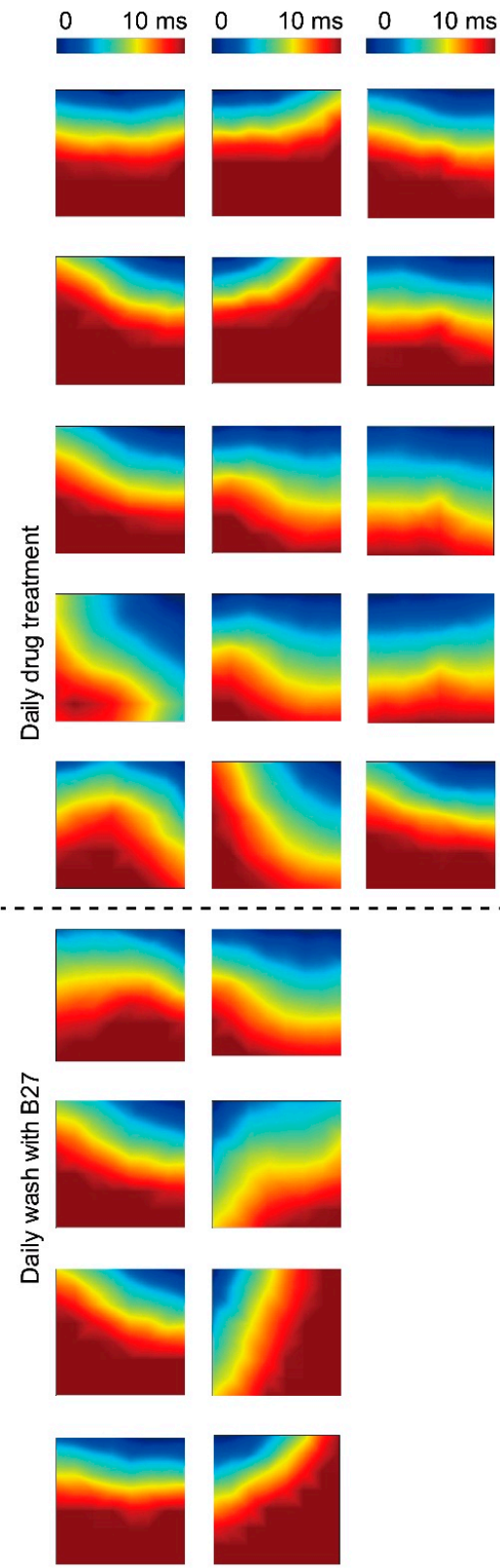

$\mathrm{B}$

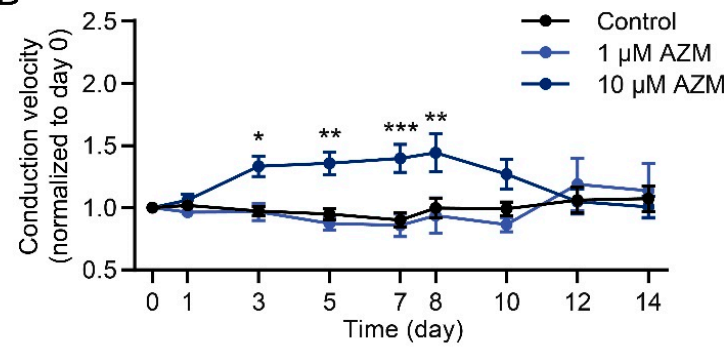

C

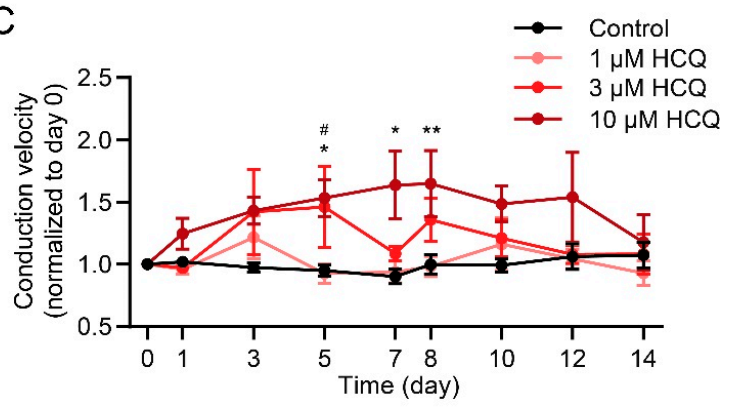

D

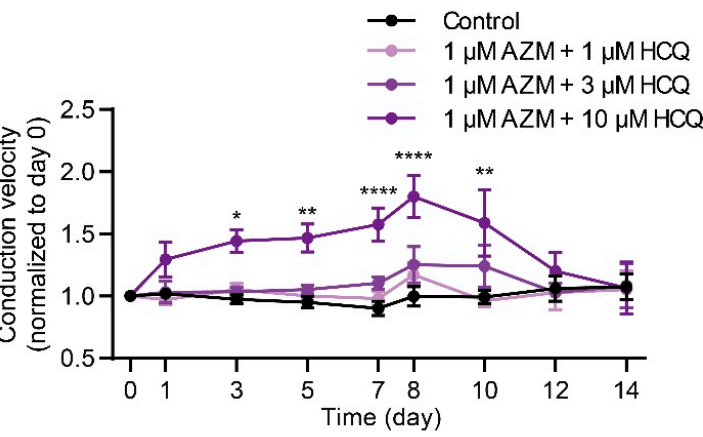

E

$\rightarrow$ Control $10 \mu \mathrm{MAZM}+1 \mu \mathrm{M} \mathrm{HCQ}$

$\rightarrow 10 \mu \mathrm{MAZM}+3 \mu \mathrm{M} \mathrm{HCQ}$

$\because 10 \mu \mathrm{MAZM}+10 \mu \mathrm{M} \mathrm{HCQ}$

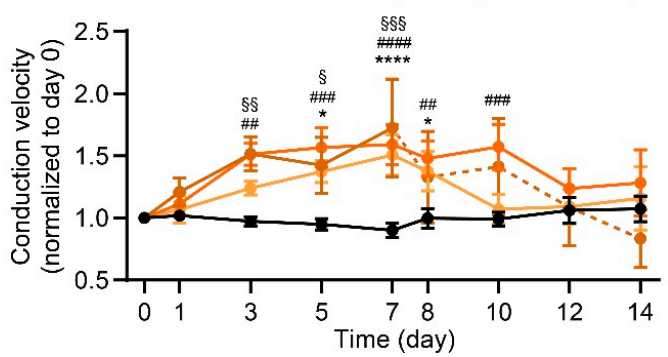

Figure 5. Changes in conduction trajectory and augmented CV in iPSC-CMs treated with AZM and HCQ alone and in combination. (A) Representative heatmaps illustrating the conduction trajectories of electrical signals in iPSC-CMs under different conditions during 7-day drug treatment and following 7-day washout. Due to cell death, no signal was captured in cells treated with $10 \mu \mathrm{M}$ HCQ and $10 \mu \mathrm{M}$ AZM in combination in the washout period. (B,C) CV of iPSC-CMs treated with AZM (B, *: $10 \mu \mathrm{M}$ AZM vs. control) and HCQ (C, \#: $3 \mu \mathrm{M}$ HCQ vs. control, *: $10 \mu \mathrm{M} \mathrm{HCQ} \mathrm{vs.} \mathrm{control)} \mathrm{for} 7$ days and following washout for 7 days (normalized to day 0 ). (D,E) CV of iPSC-CMs treated with $1 \mu \mathrm{M}(\mathbf{D}$, *: $1 \mu \mathrm{M} \mathrm{AZM}+10 \mu \mathrm{M} \mathrm{HCQ}$ vs. control) and $10 \mu \mathrm{M} \mathrm{AZM} \mathrm{(E,} \mathrm{*:} 10 \mu \mathrm{M} \mathrm{AZM}+1 \mu \mathrm{M} \mathrm{HCQ}$ vs. control, \#: $10 \mu \mathrm{M}$ AZM $+3 \mu \mathrm{M}$ HCQ vs. control, $\$: 10 \mu \mathrm{M}$ AZM $+10 \mu \mathrm{M}$ HCQ vs. control) combined with HCQ (1, 3, and $10 \mu \mathrm{M}$ ) during 7-day treatment and following 7-day washout. iPSC-CMs derived from four donors were used for MEA recording. Spontaneous beating states of iPSC-CMs are listed in Table S1. Two-way

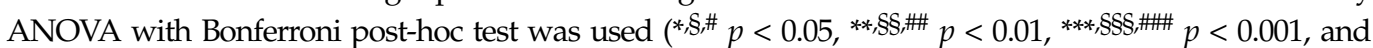
****,\#\#\# $p<0.0001)$. 
A

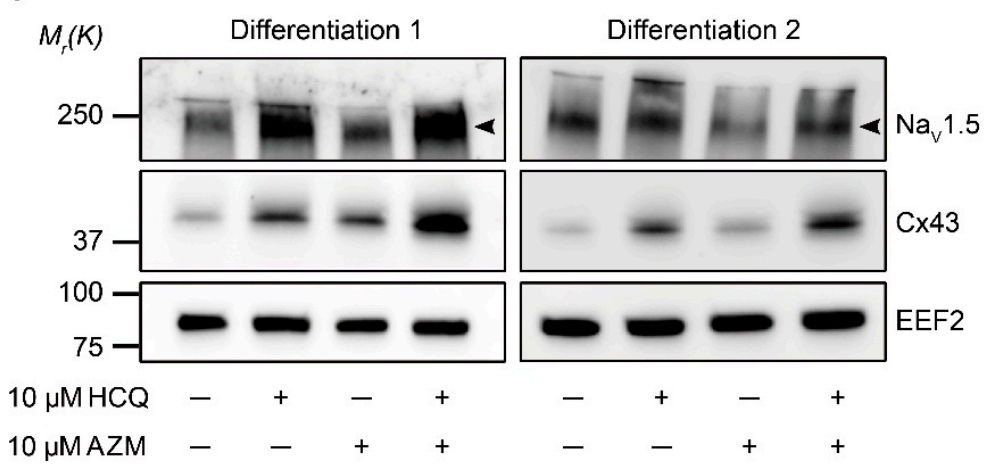

B

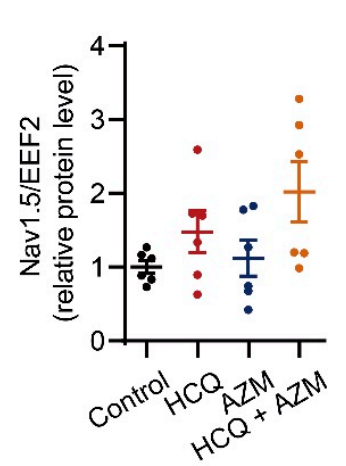

C

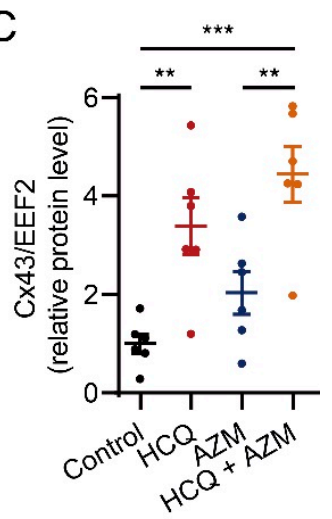

D
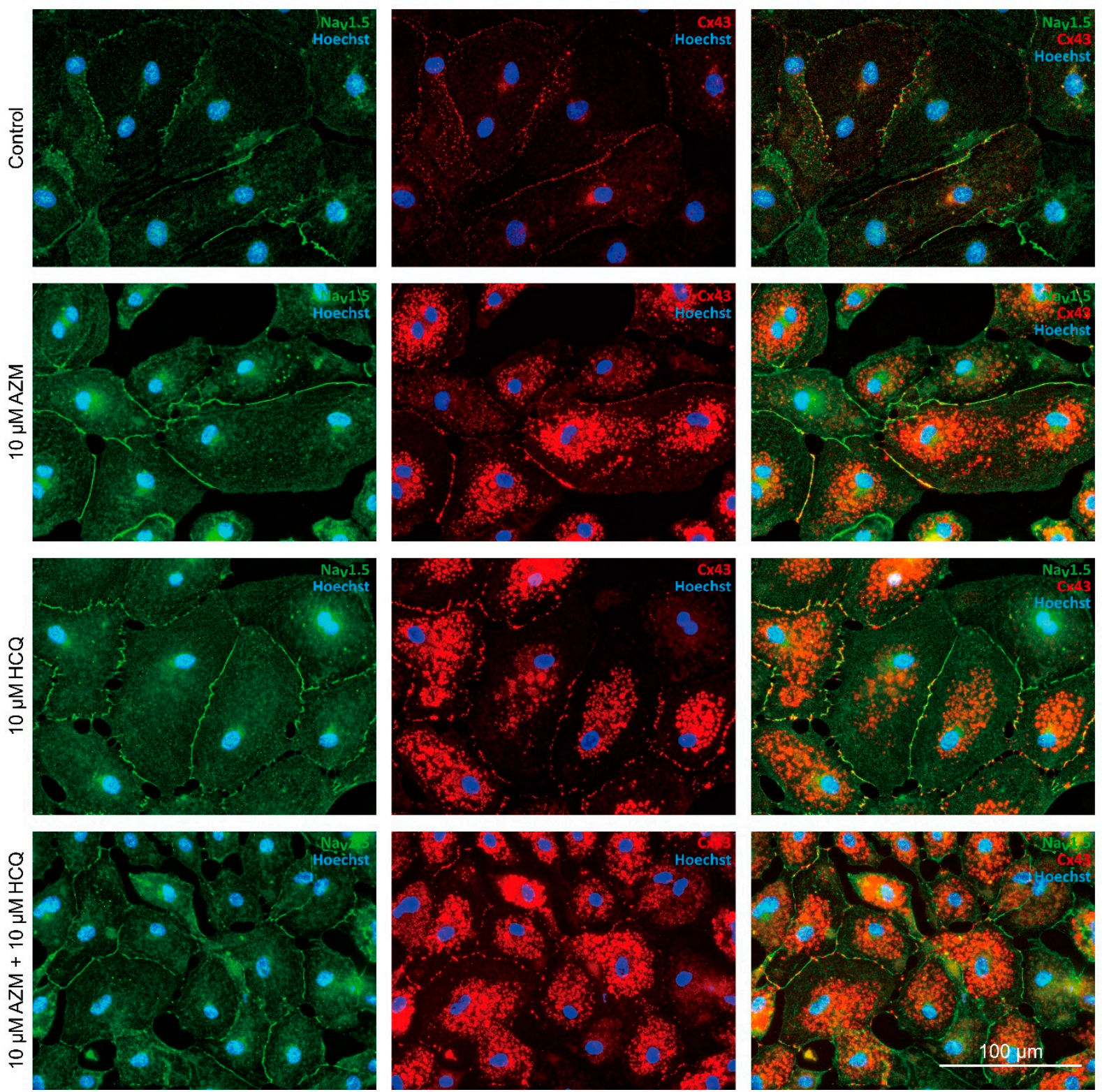

Figure 6. Nav1.5 and Cx43 protein expression in iPSC-CMs treated with HCQ and/or AZM. (A) Two representative Western blots showing the expression of Nav1.5 and Cx43 in iPSC-CMs under different drug treatment conditions. (B) Quantitation of protein expression levels of Nav1.5 in iPSC-CMs under different conditions; $N=6$ independent differentiations. (C) Quantitation of protein expression of $\mathrm{Cx} 43$ in iPSC-CMs under different drug treatment conditions; $N=6$ independent differentiations. 
(D) Representative images showing immunostaining for Nav1.5 (green) and Cx43 (red) in iPSC-CMs under different drug treatment conditions. Cell nuclei are shown in blue (Hoechst). Statistical evaluation was performed using one-way ANOVA with Tukey's multiple comparison test ${ }^{* *} p<0.01$, and $\left.{ }^{* * *} p<0.001\right)$.

A

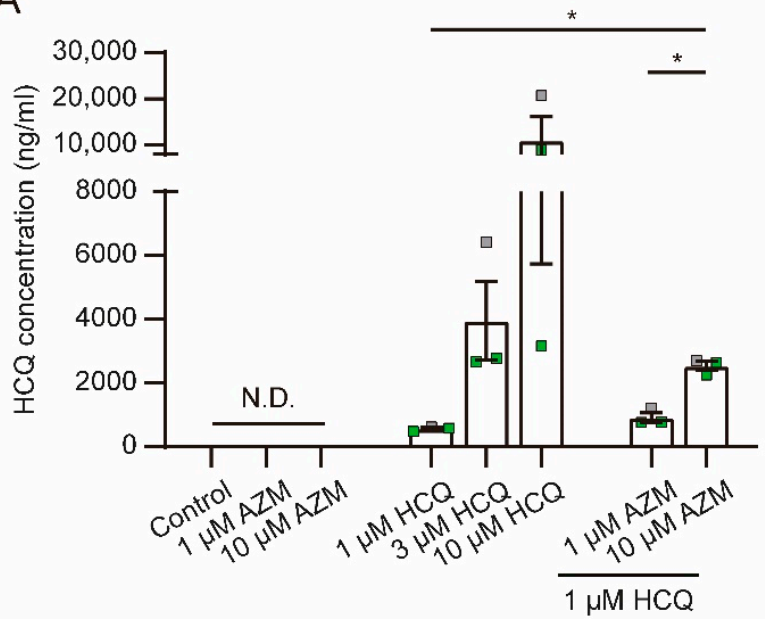

B

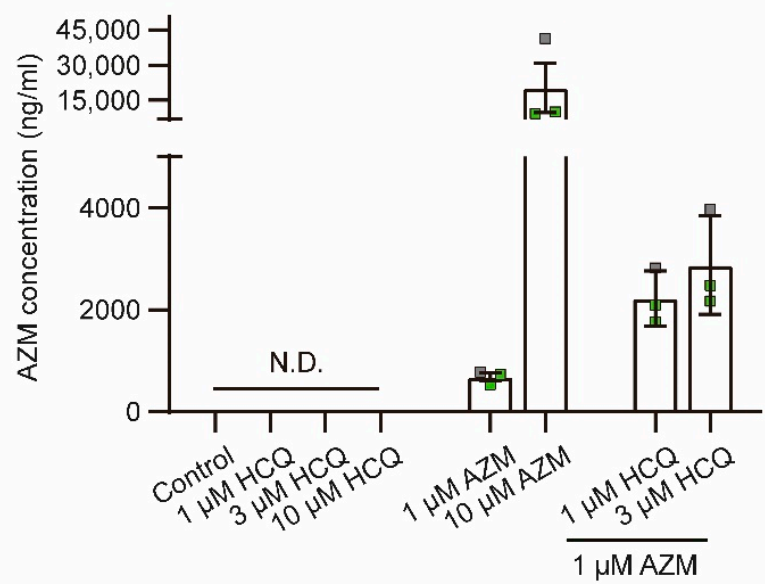

Figure 7. Accumulation of HCQ and AZM in iPSC-CMs after the 7-day treatment. (A,B) Concentrations of HCQ (A) and AZM (B) in cell lysates from iPSC-CMs after the 7-day treatment with HCQ and AZM at different conditions, determined using mass spectrometry. Data represent mean and SEM of $N=3$ independent experiments, performed with iPSC-CMs from 2 healthy donors (iBM76.1, iBM76.3 in green; iWTD2.1 in grey). N.D., below detection limit. Statistical evaluation was performed using one-way ANOVA with Tukey's multiple comparison test $\left({ }^{*} p<0.05\right)$.

\section{Discussion}

The HCQ/AZM combination therapy was associated with increased cardiac complication rates in comparison to monotherapy with HCQ or AZM [8]. Based on the known cardiac adverse effects of HCQ and AZM, and their accumulation properties, here we investigate their potential synergistic effects on iPSC-CM structure and function for a period of 7 days, similar to clinical treatment durations of $5-10$ days $[3,6,9,32,40]$.

In contrast to acute functional changes caused by inhibition of ion channels, cardiotoxic effects and reduced cell viability induced through mitochondrial dysfunction or impaired autophagy may establish during longer time scales. We show that both AZM and HCQ negatively affect the viability, morphology, and sarcomeric structure as well as the functionality of iPSC-CMs at clinically relevant concentrations and treatment duration. The progressively observed changes in contractile and electrophysiological parameters during 7 days of drug treatment, revealed by MEA-measurements and video-based motion analysis, provide first evidence for the functional consequences of AZM and HCQ under long-term treatment, whereas insights from previous studies are limited to acute or shortterm treatment $[20,21]$. Interestingly, our data reveal synergistic effects of AZM and HCQ. The combination with AZM strongly increased HCQ-induced reduction of cell viability as well as changes in contractile and electrophysiological function. Moreover, we demonstrate that HCQ and AZM increased Cx43 and Nav1.5 protein levels in a synergistic manner, which may underlie the severe electrophysiological dysfunction. Mechanistic insights on the synergistic effect of HCQ and AZM are provided by the increased accumulation of the drugs in iPSC-CMs when applied in combination.

\subsection{HCQ and AZM Differentially Affect iPSC-CM Viability and Functionality}

In this study, treatments with AZM and HCQ alone revealed that both drugs at higher concentrations negatively impact cell viability, morphology, sarcomeric structure, the contractility, and electrophysiological function of iPSC-CMs. At an equimolar concentration 
of $10 \mu \mathrm{M}$, however, a significantly higher cardiotoxic activity of HCQ than that of AZM was observed, as shown by lower MTT reduction to formazan, lower cell density, and higher LDH activity after the 7-day treatment (Figures 1 and S1A). Even after 7 days of drug washout, a progressive cardiotoxic effect of HCQ was detected not only by the MTT assay, morphological analysis, but also by the increasing the number of iPSC-CM cultures that stopped spontaneous beating (Table S1). Besides the reduced cell viability, treatment with $10 \mu \mathrm{M}$ HCQ resulted in a progressive increase in FPDc, CV, and beating frequency in iPSC-CMs during the 7-day treatment. The increased FPDc was also reported in the guinea pig heart upon acute treatment with $10 \mu \mathrm{M} H C Q$ alone ex vivo [20]. In our study, we observed a slight reduction of FPDc in iPSC-CMs after the 7-day treatment with 10 $\mu \mathrm{M}$ AZM alone, while CV was increased to a similar extend as in cells treated with $10 \mu \mathrm{M}$ HCQ. Interestingly, AZM led to an initial increase in the beating frequency on day 1, but a decrease to control levels on day 3 and a further decrease until day 7. The AZM-induced increase in the beating rate at day 1 is in line with the previous study showing that treatment of HL-1 CMs with $100 \mu \mathrm{M}$ AZM for $24 \mathrm{~h}$ dramatically increased the spontaneous beating frequency [22]. Although several studies reported the electrophysiological effects of HCQ or AZM in cardiomyocytes in vitro, our study is the first to evaluate HCQ and AZM in terms of the effect of clinically relevant long-term treatment [22].

In agreement with the reduced cell viability and impaired electrophysiological function, iPSC-CMs also showed altered contractile performance. Treatment with $10 \mu \mathrm{M} \mathrm{AZM}$ or HCQ led to decreased contraction and relaxation time as well as highly varying contraction and relaxation velocities, indicating that treatments with AZM or HCQ at a high concentration over a long time period interfere with the ability of iPSC-CMs to contract in a coordinated manner. In a recent publication, the effects of two cardiotoxic drugs, doxorubicin (DOX) and trastuzumab (TRZ), on the viability and function of iPSC-CMs were reported [41]. Unlike in our study, spontaneous beating frequency and electrical propagation of iPSC-CMs were not affected by DOX and TRZ, but the contraction velocity and displacement (or deformation distance) were reduced. These findings point toward different mechanisms of drug-induced cardiac complications induced by AZM and HCQ compared to DOX and TRZ. The adverse effects induced by DOX and TRZ were proposed to be linked to drug-induced mitochondrial dysfunction and altered cardiac energy metabolism [41]. Based on our results, we assume that the HCQ-induced increase in CV and alteration in contraction may be caused by enhanced expression (or accumulation) of Cx43 and altered gating properties of the sodium channel. Acute treatment with HCQ was reported to have an effect on $I_{N a}$ with an $\mathrm{IC}_{50}$ of $113.9 \pm 78.3 \mu \mathrm{M}$, which may explain the reduction in the electrical signal transmission observed in the guinea pig heart treated with $10 \mu \mathrm{M} H C Q$ ex vivo [20]. In our study, we observed no effect of $10 \mu \mathrm{M} H C Q$ on $I_{N a}$ after the 7-day treatment, but altered gating properties. This may account for the different effect of HCQ on CV in iPSC-CMs compared to that in the whole heart after acute treatment with $10 \mu \mathrm{M}$ HCQ. In addition, we cannot exclude the possibilities that these different effects are due to species differences between humans and guinea pigs.

It is worth mentioning that the effects of AZM and HCQ at low concentrations (1 or $3 \mu \mathrm{M}$ ) on cell area, and sarcomere structure of iPSC-CMs were relatively mild but failed to recover to the control level after 7 days of drug washout, suggesting that AZM and HCQ may induce persistent, long-term damage of iPSC-CM structure. In addition, treatment with AZM caused cellular hypertrophy, as shown by increased cell area and higher membrane capacitance (Figures 2B and S7B) whereas HCQ (1 or $3 \mu \mathrm{M})$ reduced cell area in a concentration-dependent pattern.

Overall, investigation of the individual effects of AZM and HCQ on iPSC-CMs revealed remarkable differences in their influence on the beating frequency, contractile properties, as well as FPDc. 


\subsection{Synergic Effects between $A Z M$ and $H C Q$}

Higher mortality rates, significantly increased risks for cardiac arrest [42], and greater QTc prolongation $[9,10]$ were observed in patients treated with HCQ and AZM in combination compared to treatment with either HCQ or AZM. By treating iPSC-CMs with a combination of AZM and HCQ, we confirmed this synergistic effect, which caused a strong reduction in cell viability, sarcomere disorganization, conduction abnormalities, and contractile dysfunction.

On a functional level, changes in contractile activity and electrophysiological properties induced by HCQ and AZM were more pronounced in iPSC-CMs with the combined treatment. In the presence of $10 \mu \mathrm{M} A Z M$, contraction and relaxation time was already shortened in combination with $1 \mu \mathrm{M}$ HCQ and were even more reduced with $3 \mu \mathrm{M}$ and $10 \mu \mathrm{M}$ HCQ. In addition, the HCQ-induced prolongation of FPDc was further exacerbated in the presence of $10 \mu \mathrm{M} \mathrm{AZM}$, correlating with greater QTc prolongation in patients receiving HCQ and AZM in combination [9,10]. Similar to FPDc, co-treatment with $10 \mu \mathrm{M}$ AZM was shown to potentiate the HCQ-induced increase in CV. Of note, documentations of the iPSC-CMs during the 7-day washout period revealed reversibility of the changes in FPDc and CV in some cultures. However, more iPSC-CM cultures with AZM and HCQ combination treatment showed beating arrest compared to treatment with HCQ and AZM alone. These results demonstrate that application of AZM together with HCQ worsens the adverse effects of HCQ to induce contractile and electrophysiological dysfunction in iPSC-CMs. These findings are in line with the increased expression of Nav1.5 and Cx43 in iPSC-CMs, which are induced by HCQ and AZM in a synergistic manner.

In terms of cell viability and structural organization, treatment with $10 \mu \mathrm{M} \mathrm{AZM}$ had no effect on cell viability, but the combination of $10 \mu \mathrm{M}$ HCQ with $1 \mu \mathrm{M}$ or $10 \mu \mathrm{M}$ AZM significantly enhanced the cytotoxicity of $10 \mu \mathrm{M} \mathrm{HCQ}$, as indicated by lower MTT reduction to formazan and increased LDH activity. This potential of AZM to enhance cytotoxic effects of different drugs was previously demonstrated in cancer cell lines for the combination of AZM with Lansoprazol [43] or gefitinib [44]. Similar to the viability studies, the combination of AZM with HCQ led to the most pronounced reductions in cell area, sarcomere length, and degree of sarcomeric organization, compared to the effects of AZM or HCQ alone.

\subsection{Mechanistic Evidence of AZM and HCQ Combination}

HCQ and AZM are lysosomotropic compounds known to accumulate in lysosomes and to increase lysosomal $\mathrm{pH}$, which is critical for the inhibition of viral infection [45]. Determination of drug levels in iPSC-CMs after the 7-day treatment with AZM or HCQ alone revealed that the cellular levels correlated with the drug concentrations used. Interestingly, cellular levels of AZM were higher when HCQ was present and vice versa, indicating that the combined treatment favors the accumulation of both compounds in iPSC-CMs. Previous study suggested that the ATP-dependent translocase ABCB1 plays an important role in the synergistic effects of AZM and HCQ. ABCB1 is located in the cell membrane and lysosomal membrane, and acts as an AZM-transporter and is known to be inhibited by HCQ [46]. However, involvement of ABCB1 in the synergistic effect of AZM and HCQ in iPSC-CMs is unlikely, as RNA-sequencing data from our group as well others reveal that $\mathrm{ABCB} 1$ is not expressed in iPSC-CMs $[47,48]$. So far, the mechanism for the increased cellular accumulation of AZM and HCQ with combined treatment is unclear.

Activation of integrated stress response pathway and inhibition of autophagosome formation by AZM and HCQ likely explain the strong intracellular accumulation of Nav1.5 and Cx43. Previous studies showed that application of CQ increased the abundance of $\mathrm{Cx} 43$ in neonatal rat ventricular myocytes through its lysosomal inhibiting ability and prolongation of Cx43 turnover [49,50]. Remarkably, our study shows that the synergistic effect of AZM and HCQ increased Cx43 expression by 4-fold, which was significantly higher than the increase in $\mathrm{Cx} 43$ protein expression observed by treatment with AZM alone. Additionally, 7-day treatment with AZM and HCQ increased protein expression 
of Nav1.5 but did not increase sodium current density, suggesting that the availability of functional sodium channels on the membrane was not altered despite the intracellular accumulation [22]. As cardiac conduction is determined not only by sodium channel availability but also by gap junction expression and function, our data suggest that the significantly increased expression of the gap junctional protein $\mathrm{Cx} 43$ may contribute to the increased CV in iPSC-CMs after the 7-day treatment with HCQ or HCQ and AZM in combination.

Taken together, our results reveal that the more severe effects of the combined treatment with AZM and HCQ on viability, structure, and functionality of iPSC-CMs may be caused by an increased intracellular accumulation of the drugs. The synergistic upregulation of Cx43 protein levels by AZM and HCQ provides first mechanistic evidence for the increased cardiac complications observed with the combination treatment.

\subsection{Study Limitations}

Aiming to gain mechanistic insights for the increased rates of cardiac complications observed for the combined treatment with AZM and HCQ, we characterized the consequences of the two drugs as well as their combination on the viability, structure, and functionality of iPSC-CMs. Despite human iPSC-CMs represent an important in vitro model system to study drug effects on the human heart, different aspects, including the immaturity of the cells and the lack of the multicellular environment, may influence data interpretation, particularly the susceptibility of the cells to drug concentrations. Nevertheless, we believe that human iPSC-CMs are a valuable system for predicting cardiac toxicity of the drug, relevant to human cardiac electrophysiology and dysregulation of a variety of cellular processes. In this study, we applied human iPSC-CMs from four healthy donors without known cardiovascular diseases. Modeling the situation of existing cardiac dysfunctions in patients may require the use of patient-specific iPSC-CMs to extend our study.

\section{Materials and Methods}

\subsection{Culture and Maintenance of iPSCs}

Human iPSC lines used in this study were reprogrammed from somatic cells of four healthy individuals. The cell lines iWTD2.1/2.3 (UMGi001-A clone 1 and clone 3) and iBM76.1/76.3 (UMGi005-A clone 1 and clone 3) were generated from dermal fibroblasts and mesenchymal stem cells, respectively, using STEMCCA lentivirus, and characterized as previously described [47,51]. The cell lines isWT1.13 (UMGi014-C clone 3) and isWT7.22 (UMGi020-B clone 22) were generated from dermal fibroblasts using the integration-free CytoTune-iPS 2.0 Sendai Reprogramming Kit (Thermo Fisher Scientific, Waltham, MA, USA), and characterized previously [52]. The iPSC generation was approved by the Ethics Committee of the University Medical Center Göttingen (approval number: 21/1/11 and 10/9/15) and used following the approval guidelines. To maintain the growth of iPSCs, a chemically defined E8 medium (Thermo Fisher Scientific, Waltham, MA, USA) was used, and cells were cultivated on Geltrex (Thermo Fisher Scientific, Waltham, MA, USA) coated plates at $37{ }^{\circ} \mathrm{C}$ with $5 \% \mathrm{CO}_{2}$. The E8 medium was changed on a daily basis and cells at $\sim 85 \%$ confluency were passaged using Versene (Thermo Fisher Scientific, Waltham, MA, USA).

\subsection{Differentiation of iPSCs into Cardiomyocytes and Drug Treatment}

Directed differentiation of iPSCs into cardiomyocytes was induced by modulating the WNT signaling cascade as described $[53,54]$. In brief, when iPSCs grown on 12-well plates reached 80 90\% confluency, the medium was changed from the E8 medium to cardio differentiation medium, which is composed of RPMI 1640 with Glutamax and HEPES (Thermo Fisher Scientific, Waltham, MA, USA), $0.5 \mathrm{mg} / \mathrm{mL}$ human recombinant albumin (SigmaAldrich, St. Louis, MO, USA), and $0.2 \mathrm{mg} / \mathrm{mL}$ L-ascorbic acid 2-phosphate (Sigma-Aldrich, St. Louis, MO, USA). To initiate differentiation, cells were incubated with $4 \mu \mathrm{M}$ CHIR99021 (a GSK3 $\beta$ inhibitor, Merck Millipore, Darmstadt, Germany) for $48 \mathrm{~h}$ followed by incubation with $5 \mu$ M IWP2 (a WNT signaling inhibitor, Merck Millipore, Darmstadt, Germany) for an 
additional $48 \mathrm{~h}$. Thereafter, cells were kept in cardio differentiation medium for four days with medium change every second day. The first beating cells were detected on day 8 post differentiation. From day 8, cells were cultivated in RPMI/B27 medium containing RPMI 1640 with Glutamax and HEPES, supplemented with 2\% B27 (Thermo Fisher Scientific, Waltham, MA, USA).

To maintain a long-term culture, iPSC-CMs were replated from 12-well plates into 6-well plates at day 20 post differentiation. Briefly, cells were incubated with $1 \mathrm{mg} / \mathrm{mL}$ collagenase B (Worthington Biochemical, Lakewood, USA) for $1 \mathrm{~h}$ at $37^{\circ} \mathrm{C}$. Detached iPSC-CM clusters were gently collected into a 15-mL Falcon tube and dissociated with $0.25 \%$ trypsin/EDTA (Thermo Fisher Scientific, Waltham, MA, USA) for 8 min at $37{ }^{\circ} \mathrm{C}$. Dissociated iPSC-CMs were resuspended in cardio digestion medium (80\% RPMI/B27 medium, 20\% fetal calf serum, and $2 \mu \mathrm{M}$ thiazovivin (Merck Millipore, Darmstadt, Germany)) and cultured in Geltrex-coated 6-well plates at a density of 800,000 cells per well for $24 \mathrm{~h}$. Afterward, iPSC-CMs were cultivated in RPMI/B27 medium.

To perform functional analyses, 70-day-old iPSC-CMs were dissociated again with collagenase B and trypsin stepwise, and replated for different assays. One week after replating, the cells were treated with HCQ and AZM alone or in combination at different concentrations for 7 days, with daily medium change, followed by a 7-day washout period with RPMI/B27 medium (Figure S1). HCQ (EMD Merck Millipore, Darmstadt, Germany) was dissolved in $\mathrm{ddH}_{2} \mathrm{O}$ and AZM (Sigma-Aldrich, St. Louis, MO, USA) was dissolved in DMSO to prepare $10 \mathrm{mM}$ stock solutions, which were aliquoted and stored at $-20^{\circ} \mathrm{C}$.

\subsection{Video-Based Contraction Analysis}

Video-based analyses were used to examine drug effects on the contractile parameters of iPSC-CMs. To this end, iPSC-CMs were replated into Geltrex-coated 48-well plates at a density of 60,000 cells per well one week before drug treatment. Videos were obtained using an ORCA Flash 4.0 V3 CMOS camera (Hamamatsu Photonics, Hamamatsu, Japan, 60 FPS, $1024 \times 1024$ pixels resolution) on days 0 (right before treatment), 1, 3, 5, and 7 of the treatment. Video data were analyzed using the cellular motion analysis software "Maia" (QuoData-Quality \& Statistics $\mathrm{GmbH}$ ) to evaluate the beating properties [55]. Analysis settings were: block size $20.3 \mu \mathrm{m}$ (16 pixels), frameshift $100 \mathrm{~ms}$, and maximum distance shift $8.9 \mu \mathrm{m}$ (7 pixels). For every condition, videos were obtained from three different wells with two videos on different areas of each well. For analysis, data were normalized to control without drugs of the respective day.

\subsection{Immunofluorescence Staining}

For immunostainings, iPSC-CMs were seeded into Geltrex-coated 12-well or 6-well plates prepared with coverslips at a density of 15,000 or 200,000 cells per well, respectively. After seeding, cells were cultured for 7 days in RPMI/B27 medium before drug treatment. On day 7 (after drug treatment for 7 days) or day 14 (after drug washout for 7 days), cells were washed 2 times for 5 min in relaxation buffer (PBS supplemented with 5 mM EGTA and $5 \mathrm{mM} \mathrm{MgCl} 2$ ), followed by 2 times wash with PBS and fixation in ice-cold methanol-acetone $(7: 3, v / v)$ solution for $20 \mathrm{~min}$ at $-20^{\circ} \mathrm{C}$. Fixed cells were washed 3 times for $5 \mathrm{~min}$ with PBS, followed by blocking in 1\% BSA (bovine serum albumin) for at least $2 \mathrm{~h}$ at $4{ }^{\circ} \mathrm{C}$. For staining, cells were incubated with the following primary antibodies: anti- $\alpha$-actinin (clone EA-53; 1:500; mouse monoclonal, IgG1, Sigma-Aldrich, St. Louis, MO, USA, 7811), antiNav1.5 (1:200; rabbit polyclonal, Alomone Labs, Jerusalem, Israel, ASC-005), and anti-Cx43 (clone 2; 1:1000; mouse monoclonal, IgG1, BD Biosciences, Franklin Lakes, USA, 610061) at $4{ }^{\circ} \mathrm{C}$ overnight. Afterward, cells were washed three times with PBS and incubated with the corresponding secondary antibodies (1:1000; anti-rabbit Alexa Fluor 488, Invitrogen, Waltham, MA, USA, A11008; anti-mouse Alexa Fluor 488, Invitrogen, Waltham, MA, USA, A11001; or anti-mouse Alexa Fluor 546, Invitrogen, Waltham, MA, USA, A11030) for $1 \mathrm{~h}$ at room temperature. Cell nuclei were counterstained with Hoechst33342 (1:1000; Thermo Fisher Scientific, Waltham, MA, USA) in PBS for $20 \mathrm{~min}$. Coverslips were mounted on 
glass slides using Fluoromount-G mounting medium (Thermo Fisher Scientific, Waltham, MA, USA). Stained iPSC-CMs were imaged using a fluorescence microscope (Keyence BZ-X700E, Keyence, Osaka, Japan). Quantification of cell area was performed in iPSC-CMs stained for $\alpha$-actinin using Cell Profiler [56] and manual analysis with FIJI [57]. Sarcomere length was determined manually using FIJI as described previously [36]. The amount of structurally organized iPSC-CMs with evenly distributed intact sarcomeres across the cell body (occupying $>80 \%$ of the cell area) and disorganized cells was determined using manual counting.

\subsection{Multi-Electrode Array}

For FP measurement, iPSC-CMs were seeded in the cavity containing electrodes of the Geltrex-coated CytoView 6-well MEA plates (Axion BioSystems, Atlanta, GA, USA). Around 300,000 iPSC-CMs were resuspended in $20 \mu \mathrm{L}$ cardio digestion medium and seeded in the electrode-containing cavity of the MEA plates. One hour later, an additional $1 \mathrm{~mL}$ of medium was added into each well. iPSC-CMs were kept in RPMI/B27 medium for one week before drug treatment. For every batch of experiment, at least two wells of iPSCCMs from different plates were treated with the same condition to avoid plate variability. Spontaneous FP recordings were carried out using the Maestro Edge equipped with AxIS Navigator software (Axion BioSystems, Atlanta, GA, USA) with a sample rate of 12,500 Hz at $37^{\circ} \mathrm{C}$ with $5 \% \mathrm{CO}_{2}$. From day 0 (right before treatment) to day 14 (last day for washout), FPs were recorded daily for all conditions used (Figure S1). Several key parameters including conduction velocity $(\mathrm{CV})$, corrected FPD $_{\mathrm{C}}$ (corrected by Fridericia's formula), and inter-beat interval were determined using AxIS Navigator, and further analyzed with AxIS Metric Plotting Tool (Axion BioSystems, Atlanta, GA, USA). Spontaneous beating frequency was defined as the reciprocal of averaged inter-beat interval. The mainstream $\mathrm{CV}$ values were averaged for one culture.

\subsection{Automated Patch-Clamp}

To investigate the effect of high concentrations of HCQ and AZM on the function of sodium channel, the properties of $I_{\mathrm{Na}}$ were examined in iPSC-CMs treated with $10 \mu \mathrm{M} \mathrm{HCQ}$ alone, $10 \mu \mathrm{M}$ AZM alone, or their combination, respectively. The drug treatment lasted for 7 days with daily medium change, and iPSC-CMs kept in RPMI/B27 medium served as control. Recording of $I_{\mathrm{Na}}$ was performed using the Patchliner Quattro (Nanion Technologies $\mathrm{GmbH}$, Munich, Germany) with low resistance NPC-16 chips at room temperature as described previously [53,58,59]. In brief, iPSC-CMs were dissociated gently into single cells. Capture of single cells and formation of whole-cell configuration were processed automatically by Patchliner. From a holding potential of $-100 \mathrm{mV}, I_{\mathrm{Na}}$ was recorded under pulses ranging from -90 to $+70 \mathrm{mV}$ for $20 \mathrm{~ms}$ in $5 \mathrm{mV}$ increment with an interval of $2 \mathrm{~s}$. Currents were sampled at $25 \mathrm{kHz}$ and low-pass-filtered at $2.9 \mathrm{kHz}$.

\subsection{Western Blot}

Three-month-old iPSC-CMs were treated with $10 \mu \mathrm{M}$ HCQ, or $10 \mu \mathrm{M}$ AZM, or the combination of HCQ and AZM for seven days, snap-frozen in liquid nitrogen and stored at $-80{ }^{\circ} \mathrm{C}$. To detect the expression of specific proteins, cells were lysed by homogenization in RIPA buffer (150 mM NaCl, 50 mM Tris, 1.0\% NP-40, 0.5\% sodium deoxycholate, 0.1\% SDS, $1 \mathrm{mM}$ EDTA, $10 \mathrm{mM} \mathrm{NaF}$, and $1 \mathrm{mM}$ PMSF), supplemented with protease (cOmplete mini, EDTA-free, Roche, Basel, Switzerland) and phosphatase (PhosSTOP, Roche, Basel, Switzerland) inhibitors and incubated for $30 \mathrm{~min}$ at $4{ }^{\circ} \mathrm{C}$ with gentle rotation. Cell homogenates were clarified by centrifugation at $14,000 \mathrm{rpm}$ for $20 \mathrm{~min}$ at $4{ }^{\circ} \mathrm{C}$ and protein concentration was measured using a BCA assay following the manufacturer's instruction. Proteins (30 $\mu \mathrm{g} /$ sample) were subjected to SDS-PAGE using a 4-15\% gradient gel (BioRad, Hercules, CA, USA) and transferred onto nitrocellulose membranes. Membranes were blocked in 5\% milk in TBS-T for 30-45 min at room temperature and probed with anti-Cx43 (clone 4E6.2; 1:1000; mouse monoclonal, Merck Millipore, Darmstadt, Germany, MAB3067), anti-Nav1.5 
(1:200; rabbit polyclonal, Alomone Labs, Jerusalem, Israel, ASC-005), or anti-EEF2 (1:5000; rabbit polyclonal, Abcam, Cambridge, UK, ab40812) antibodies at $4{ }^{\circ} \mathrm{C}$ overnight, followed by incubation with horseradish peroxidase-conjugated secondary antibodies goat antimouse (1:10,000; Sigma Aldrich, St. Louis, MO, USA, A2304) or goat anti-rabbit (1:10,000; Cell Signaling, Danvers, MA, USA, 7074S) for $1 \mathrm{~h}$ at room temperature. Proteins were visualized by chemiluminescence using the Super Signal West Dura Chemiluminescent Substrate kit in combination with the Fusion FX Spectra Imaging System (Peqlab, VWR, Radnor, PA, USA). Densitometry analyses of the immunoblots were performed using ImageJ software and the intensity of individual bands was normalized to EEF2.

\subsection{Lactate Dehydrogenase Measurement}

Measurement of LDH activity was performed using LDH assay kit (Abcam, Cambridge, UK, ab102526) according to the manufacturer's instructions in supernatants of iPSC-CM cultures after 7 days of drug treatment and after subsequent 7 days of drug washout. Briefly, $50 \mu \mathrm{L}$ of cell supernatant was mixed with $50 \mu \mathrm{L}$ substrate solution in a 96 well plate. Absorption was measured at $450 \mathrm{~nm}$ in a kinetic mode, every $2 \mathrm{~min}$ for $60 \mathrm{~min}$ (Biotek Synergy HTX, Biotek Instruments-Agilent, Santa Clara, CA, USA). LDH activity was calculated based on a standard curve according to the manufacturer's instructions (Equation (1)).

Equation (1): Calculation of LDH activity

LDH activity $\left[\frac{\mathrm{mU}}{\mathrm{mL}}\right]=\left(\frac{\text { Amount of } N A D H \text { in sample calc. from standard curve }[\mathrm{nmol}]}{\text { reaction time }[\mathrm{min}] \times \text { Sample volume }[\mathrm{mL}]}\right) \times$ Dilution factor

\subsection{MTT Assay}

Cell viability was determined using MTT assay kit (Merck Millipore, Darmstadt, Germany, CT02) according to the manufacturer's instructions. After drug treatment as well as after drug washout, cells were washed twice with pre-warmed PBS and incubated in $200 \mu \mathrm{L}$ RPMI/B27 medium per well with $0.5 \mathrm{mg} / \mathrm{mL}$ MTT for $2 \mathrm{~h}$ at $37^{\circ} \mathrm{C}$. Subsequently, $300 \mu \mathrm{L}$ of isopropanol with $0.04 \mathrm{~N} \mathrm{HCl}$ was added and samples were mixed thoroughly by pipetting to facilitate cell lysis and the dissolving of formazan. Absorbance was measured at $570 \mathrm{~nm}$ (formazan) and $630 \mathrm{~nm}$ (reference) using plate reader (Biotek Synergy HTX, Biotek Instruments-Agilent, Santa Clara, CA, USA). Viability was calculated as $\mathrm{A}_{570}-\mathrm{A}_{630}$.

\subsection{Determination of HCQ and AZM Concentrations in Cell Lysates}

Intracellular drug accumulation was determined from cell lysates of the MTT assay using mass spectrometry. After MTT measurement, cell lysates were stored at $-20{ }^{\circ} \mathrm{C}$ for 1-4 days prior to detection. The stability of HCQ and AZM under these conditions was confirmed for up to 7 days at $-20^{\circ} \mathrm{C}$. Total of $25 \mu \mathrm{L}$ of fresh or thawed cell lysates were diluted with $225 \mu \mathrm{L}$ of $2 \mathrm{mM}$ ammonium acetate buffer, vortexed and centrifuged for $10 \mathrm{~min}(14,000 \mathrm{rpm})$. About $10 \mu \mathrm{L}$ of the clear supernatants were injected into the LC-MS/MS, which consists of an UltiMate3000 pump, an autosampler (Dionex, ThermoScientific, St. Louis, MO, USA) and an API 4000 Tandem mass spectrometer (ABSciex, Darmstadt, Germany) using positive electrospray ionization (ESI+; $4500 \mathrm{~V})$. HCQ and AZM were determined by a Synergi $4 \mu$ HydroRP 80 A column $150 \mathrm{~mm} \times 3.0 \mathrm{~mm}$ (Phenomenex, Torrance, CA, USA) using a binary gradient with $2 \mathrm{mM}$ ammonium acetate buffer and acetonitrile and a flow rate of $0.5 \mathrm{~mL} / \mathrm{min}$. The resulting retention times were $3.0 \mathrm{~min}$ for HCQ and 3.2 min for AZM. HCQ and AZM were measured using the multiple reaction monitoring mode with nitrogen as collision gas. The method was suitable for the quantification of HCQ and AZM in cell lysates over the range from 20 to $1000 \mathrm{ng} / \mathrm{mL}$. Samples with higher concentrations were diluted. 


\subsection{Statistics}

Results about cell area are presented as median $\pm 95 \% \mathrm{CI}$ and results for the other parameters are presented as mean \pm standard error of the mean (SEM). Statistical analysis was performed with GraphPad Prism 9. One-way ANOVA with Tukey's multiple comparison was used for cell viability, cell area, sarcomere length, contractility property, protein expression level, and drug accumulation data. Two-way ANOVA with Bonferroni post-hoc test was used for MEA assay-based FPDc, CV and beating rate data, as well as Patchliner assay-based $I_{\mathrm{Na}}$ data. $p<0.05$ was considered statistically significant.

\section{Conclusions}

Through the systematic investigation of the effects of AZM and HCQ individually as well as in combination, we show that these two drugs have adverse effects on the viability, structure, and functionality of human cardiomyocytes using the in vitro iPSC-CM system. These adverse effects get more severe when AZM and HCQ are applied in combination, thus recapitulating the higher rates of cardiac complications observed with the AZM/HCQ combination treatment in clinical use. The synergistic adverse effects of AZM and HCQ in iPSC-CMs are likely driven by the increased intracellular accumulation of the drugs when applied in combination. Furthermore, we provide evidence that the HCQ-induced increase in conduction velocity might be caused by elevated levels of $\mathrm{Cx} 43$, which further increase in combination with AZM.

Supplementary Materials: The following supporting information can be downloaded at: https: / / www.mdpi.com/article/10.3390/ph15020220/s1. Figure S1: Experimental scheme. Figure S2: Morphological changes induced by treatment with HCQ and AZM, and quantification of nucleus number after 7-day treatment with the drugs. Figure S3: Cell viability and contractility in vehicle (0.1\% DMSO) treated iPSC-CMs. Figure S4: Effects of AZM and HCQ treatments on contraction parameters. Figure S5: Representative images of iPSC-CMs on MEA plate. Figure S6: Effects of HCQ and AZM treatments on the beating rates of iPSC-CMs. Figure S7: Effects of HCQ and AZM on the sodium channel. Table S1: Spontaneously beating status of iPSC-CM culture during 15 days of the recording. Video S1: Contractile behavior of iPSC-CMs after 3 days of treatment. Video S2: Contractile behavior of iPSC-CMs after 7 days of treatment.

Author Contributions: Study conceptualization by W.L., X.L., K.N., S.U., M.S. (Mario Schubert) and K.G.; supervision was performed by S.U. and K.G.; and project administration by K.H., S.U., K.S., M.S. (Mario Schubert) and K.G.; W.L., X.L., M.S.P., A.S., R.O., L.C. and M.S. (Mario Schubert) conducted investigation; and W.L., X.L., M.S.P., A.S., R.O., K.N., M.H., R.-P.S., M.S. (Mario Schubert) and K.G. performed data curation and formal analysis. W.L., X.L., K.H., S.U., M.S. (Mario Schubert) and K.G. contributed to the validation and interpretation of the data. Software was developed by K.N., M.S. (Martin Schneider), K.H. and S.U.; S.U., K.S. and K.G. acquired funding; and S.U., K.S. and K.G. provided resources. The original draft was prepared by W.L., X.L., R.O., M.S.P., M.S. (Mario Schubert) and K.G.; and finalized by W.L., M.S. (Mario Schubert) and K.G. All authors have read and agreed to the published version of the manuscript.

Funding: The work was supported by the Free State of Saxony and the European Union EFRE (SAB projects "PhänoKard" and "PhenoCor" to K. Guan as well as "HERMES" to QuoData-Quality \& Statistics GmbH (K. Simon and S. Uhlig) and to K. Guan), by the German Federal Ministry of Education and Research/German Center for Cardiovascular Research (to L. Cyganek), and by the German Research Foundation (Project Number 193793266-SFB1002 S01, to L. Cyganek). M. Schubert was supported by the MeDDrive START grant from the Medical Faculty at TU Dresden. A. Strano and M. Hasse were financially supported by the Deutsche Forschungsgemeinschaft (DFG, German Research Foundation)-Project Number 288034826-IRTG 2251: “Immunological and Cellular Strategies in Metabolic Disease".

Institutional Review Board Statement: Not applicable.

Informed Consent Statement: Not applicable.

Data Availability Statement: Data is contained within the article or Supplementary Material. 
Acknowledgments: We thank Konstanze Fischer, Ying Ulbricht, Judith Müller, Jessie Pöche, and Julian Leefmann (Dresden) for their excellent technical assistance.

Conflicts of Interest: The authors declare no conflict of interest.

\begin{abstract}
Abbreviations
ABCB1: ATP binding cassette subfamily B member 1; AZM: azithromycin; COVID-19 patients: SARS-CoV-2 infected patients; CV: conduction velocity; Cx43: connexin-43; DMSO: dimethylsulfoxide; DOX: doxorubicin; EEF2: eukaryotic elongation factor 2; FP: field potential; FPDc: corrected field potential duration; HCQ: hydroxychloroquine; iPSC: induced pluripotent stem cell; iPSC-CMs: induced pluripotent stem cell-derived cardiomyocytes; LDH: lactate dehydrogenase; MEA: multielectrode array; MTT: 3-(4,5-dimethylthiazol-2-yl)-2,5-diphenyltetrazolium bromide; Nav1.5: sodium channel, voltage-gated, type 5, alpha subunit; SARS-CoV-2: severe acute respiratory syndrome coronavirus 2; TRZ: trastuzumab.
\end{abstract}

\title{
References
}

1. Mamoshina, P.; Rodriguez, B.; Bueno-Orovio, A. Toward a broader view of mechanisms of drug cardiotoxicity. Cell Rep. Med. 2021, 2, 100216. [CrossRef] [PubMed]

2. Ma, W.; Wei, S.; Zhang, B.; Li, W. Molecular Mechanisms of Cardiomyocyte Death in Drug-Induced Cardiotoxicity. Front. Cell Dev. Biol. 2020, 8, 434. [CrossRef]

3. Gautret, P.; Lagier, J.C.; Parola, P.; Hoang, V.T.; Meddeb, L.; Mailhe, M.; Doudier, B.; Courjon, J.; Giordanengo, V.; Vieira, V.E.; et al. Hydroxychloroquine and azithromycin as a treatment of COVID-19: Results of an open-label non-randomized clinical trial. Int. J. Antimicrob. Agents 2020, 56, 105949. [CrossRef] [PubMed]

4. Andreani, J.; Le Bideau, M.; Duflot, I.; Jardot, P.; Rolland, C.; Boxberger, M.; Wurtz, N.; Rolain, J.-M.; Colson, P.; La Scola, B.; et al. In vitro testing of combined hydroxychloroquine and azithromycin on SARS-CoV-2 shows synergistic effect. Microb. Pathog. 2020, 145, 104228. [CrossRef]

5. Dixit, S.B.; Zirpe, K.G.; Kulkarni, A.P.; Chaudhry, D.; Govil, D.; Mehta, Y.; Jog, S.A.; Khatib, K.I.; Pandit, R.A.; Samavedam, S.; et al. Current Approaches to COVID-19: Therapy and Prevention. Indian J. Crit. Care Med. 2020, 24, 838-846. [CrossRef]

6. Furtado, R.H.M.; Berwanger, O.; Fonseca, H.A.; Correa, T.D.; Ferraz, L.R.; Lapa, M.G.; Zampieri, F.G.; Veiga, V.C.; Azevedo, L.C.P.; Rosa, R.G.; et al. Azithromycin in addition to standard of care versus standard of care alone in the treatment of patients admitted to the hospital with severe COVID-19 in Brazil (COALITION II): A randomised clinical trial. Lancet 2020, 396, 959-967. [CrossRef]

7. Geleris, J.; Sun, Y.; Platt, J.; Zucker, J.; Baldwin, M.; Hripcsak, G.; Labella, A.; Manson, D.K.; Kubin, C.; Barr, R.G.; et al. Observational Study of Hydroxychloroquine in Hospitalized Patients with COVID-19. N. Engl. J. Med. 2020, 382, 2411-2418. [CrossRef]

8. Chorin, E.; Dai, M.; Shulman, E.; Wadhwani, L.; Bar-Cohen, R.; Barbhaiya, C.; Aizer, A.; Holmes, D.; Bernstein, S.; Spinelli, M.; et al. The QT interval in patients with COVID-19 treated with hydroxychloroquine and azithromycin. Nat. Med. 2020, 26, 808-809. [CrossRef] [PubMed]

9. Mercuro, N.J.; Yen, C.F.; Shim, D.J.; Maher, T.R.; McCoy, C.M.; Zimetbaum, P.J.; Gold, H.S. Risk of QT Interval Prolongation Associated With Use of Hydroxychloroquine With or Without Concomitant Azithromycin Among Hospitalized Patients Testing Positive for Coronavirus Disease 2019 (COVID-19). JAMA Cardiol. 2020, 5, 1036-1041. [CrossRef]

10. Saleh, M.; Gabriels, J.; Chang, D.; Kim, B.S.; Mansoor, A.; Mahmood, E.; Makker, P.; Ismail, H.; Goldner, B.; Willner, J.; et al. Effect of Chloroquine, Hydroxychloroquine, and Azithromycin on the Corrected QT Interval in Patients With SARS-CoV-2 Infection. Circ. Arrhythm. Electrophysiol. 2020, 13, e008662. [CrossRef]

11. Ramireddy, A.; Chugh, H.; Reinier, K.; Ebinger, J.; Park, E.; Thompson, M.; Cingolani, E.; Cheng, S.; Marban, E.; Albert, C.M.; et al. Experience With Hydroxychloroquine and Azithromycin in the Coronavirus Disease 2019 Pandemic: Implications for QT Interval Monitoring. J. Am. Heart Assoc. 2020, 9, e017144. [CrossRef] [PubMed]

12. Yang, Y.P.; Hu, L.F.; Zheng, H.F.; Mao, C.J.; Hu, W.D.; Xiong, K.P.; Wang, F.; Liu, C.F. Application and interpretation of current autophagy inhibitors and activators. Acta Pharmacol. Sin. 2013, 34, 625-635. [CrossRef] [PubMed]

13. Wang, X.; Pan, Z.; Wang, J.; Wang, H.; Fan, H.; Gong, T.; Sun, Q.; Feng, Y.; Liang, P. Characterization of the molecular mechanisms underlying azithromycin-induced cardiotoxicity using human-induced pluripotent stem cell-derived cardiomyocytes. Clin. Transl. Med. 2021, 11, e549. [CrossRef] [PubMed]

14. Chatre, C.; Roubille, F.; Vernhet, H.; Jorgensen, C.; Pers, Y.-M. Cardiac Complications Attributed to Chloroquine and Hydroxychloroquine: A Systematic Review of the Literature. Drug Saf. 2018, 41,919-931. [CrossRef]

15. Roden, D.M.; Harrington, R.A.; Poppas, A.; Russo, A.M. Considerations for Drug Interactions on QTc in Exploratory COVID-19 Treatment. Circulation 2020, 141, e906-e907. [CrossRef] 
16. Capel, R.A.; Herring, N.; Kalla, M.; Yavari, A.; Mirams, G.R.; Douglas, G.; Bub, G.; Channon, K.; Paterson, D.J.; Terrar, D.A.; et al. Hydroxychloroquine reduces heart rate by modulating the hyperpolarization-activated current If: Novel electrophysiological insights and therapeutic potential. Heart Rhythm 2015, 12, 2186-2194. [CrossRef]

17. Huang, B.H.; Wu, C.H.; Hsia, C.P.; Chen, C.Y. Azithromycin-induced torsade de pointes. Pacing Clin. Electrophysiol. 2007, 30, 1579-1582. [CrossRef]

18. Ray, W.A.; Murray, K.T.; Hall, K.; Arbogast, P.G.; Stein, C.M. Azithromycin and the Risk of Cardiovascular Death. N. Engl. J. Med. 2012, 366, 1881-1890. [CrossRef]

19. Kezerashvili, A.; Khattak, H.; Barsky, A.; Nazari, R.; Fisher, J.D. Azithromycin as a cause of QT-interval prolongation and torsade de pointes in the absence of other known precipitating factors. J. Interv. Card. Electrophysiol. 2007, 18, 243-246. [CrossRef]

20. Wang, G.; Lu, C.-J.; Trafford, A.W.; Tian, X.; Flores, H.M.; Maj, P.; Zhang, K.; Niu, Y.; Wang, L.; Du, Y.; et al. Mechanistic insights into ventricular arrhythmogenesis of hydroxychloroquine and azithromycin for the treatment of COVID-19. bioRxiv 2020, 2020.2005.2021.108605. [CrossRef]

21. Yanagida, S.; Satsuka, A.; Hayashi, S.; Ono, A.; Kanda, Y. Comprehensive Cardiotoxicity Assessment of COVID-19 Treatments Using Human-Induced Pluripotent Stem Cell-Derived Cardiomyocytes. Toxicol. Sci. 2021, 183, 227-239. [CrossRef] [PubMed]

22. Yang, Z.; Prinsen, J.K.; Bersell, K.R.; Shen, W.; Yermalitskaya, L.; Sidorova, T.; Luis, P.B.; Hall, L.; Zhang, W.; Du, L.; et al Azithromycin Causes a Novel Proarrhythmic Syndrome. Circ. Arrhythm. Electrophysiol. 2017, 10, e003560. [CrossRef]

23. Touret, F.; Gilles, M.; Barral, K.; Nougairede, A.; van Helden, J.; Decroly, E.; de Lamballerie, X.; Coutard, B. In vitro screening of a FDA approved chemical library reveals potential inhibitors of SARS-CoV-2 replication. Sci. Rep. 2020, 10, 13093. [CrossRef] [PubMed]

24. Durcan, L.; Clarke, W.A.; Magder, L.S.; Petri, M. Hydroxychloroquine Blood Levels in Systemic Lupus Erythematosus: Clarifying Dosing Controversies and Improving Adherence. J. Rheumatol. 2015, 42, 2092-2097. [CrossRef]

25. Yao, X.; Ye, F.; Zhang, M.; Cui, C.; Huang, B.; Niu, P.; Liu, X.; Zhao, L.; Dong, E.; Song, C.; et al. In Vitro Antiviral Activity and Projection of Optimized Dosing Design of Hydroxychloroquine for the Treatment of Severe Acute Respiratory Syndrome Coronavirus 2 (SARS-CoV-2). Clin. Infect. Dis. 2020, 71, 732-739. [CrossRef] [PubMed]

26. Jeong, B.H.; Jeon, K.; Park, H.Y.; Moon, S.M.; Kim, S.Y.; Lee, S.Y.; Shin, S.J.; Daley, C.L.; Koh, W.J. Peak Plasma Concentration of Azithromycin and Treatment Responses in Mycobacterium avium Complex Lung Disease. Antimicrob. Agents Chemother. 2016, 60, 6076-6083. [CrossRef]

27. Zuckerman, J.M. The newer macrolides: Azithromycin and clarithromycin. Infect. Dis. Clin. N. Am. 2000, 14, 449-462. [CrossRef]

28. Tett, S.E.; Cutler, D.J.; Day, R.O.; Brown, K.F. A dose-ranging study of the pharmacokinetics of hydroxy-chloroquine following intravenous administration to healthy volunteers. Br. J. Clin. Pharmacol. 1988, 26, 303-313. [CrossRef]

29. Zheng, S.; Matzneller, P.; Zeitlinger, M.; Schmidt, S. Development of a population pharmacokinetic model characterizing the tissue distribution of azithromycin in healthy subjects. Antimicrob. Agents Chemother. 2014, 58, 6675-6684. [CrossRef]

30. Liu, J.; Cao, R.; Xu, M.; Wang, X.; Zhang, H.; Hu, H.; Li, Y.; Hu, Z.; Zhong, W.; Wang, M. Hydroxychloroquine, a less toxic derivative of chloroquine, is effective in inhibiting SARS-CoV-2 infection in vitro. Cell Discov. 2020, 6, 16. [CrossRef]

31. Araujo, F.G.; Shepard, R.M.; Remington, J.S. In vivo activity of the macrolide antibiotics azithromycin, roxithromycin and spiramycin against Toxoplasma gondii. Eur. J. Clin. Microbiol. Infect. Dis. 1991, 10, 519-524. [CrossRef]

32. Cavalcanti, A.B.; Zampieri, F.G.; Rosa, R.G.; Azevedo, L.C.P.; Veiga, V.C.; Avezum, A.; Damiani, L.P.; Marcadenti, A.; Kawano-Dourado, L.; Lisboa, T.; et al. Hydroxychloroquine with or without Azithromycin in Mild-to-Moderate COVID-19. N. Engl. J. Med. 2020, 383, 2041-2052. [CrossRef] [PubMed]

33. O'Laughlin, J.P.; Mehta, P.H.; Wong, B.C. Life Threatening Severe QTc Prolongation in Patient with Systemic Lupus Erythematosus due to Hydroxychloroquine. Case Rep. Cardiol. 2016, 2016, 4626279. [CrossRef]

34. Chen, C.Y.; Wang, F.L.; Lin, C.C. Chronic hydroxychloroquine use associated with QT prolongation and refractory ventricular arrhythmia. Clin. Toxicol. 2006, 44, 173-175. [CrossRef] [PubMed]

35. Karbassi, E.; Fenix, A.; Marchiano, S.; Muraoka, N.; Nakamura, K.; Yang, X.; Murry, C.E. Cardiomyocyte maturation: Advances in knowledge and implications for regenerative medicine. Nat. Rev. Cardiol. 2020, 17, 341-359. [CrossRef] [PubMed]

36. Kolanowski, T.J.; Busek, M.; Schubert, M.; Dmitrieva, A.; Binnewerg, B.; Poche, J.; Fisher, K.; Schmieder, F.; Grunzner, S.; Hansen, S.; et al. Enhanced structural maturation of human induced pluripotent stem cell-derived cardiomyocytes under a controlled microenvironment in a microfluidic system. Acta Biomater. 2020, 102, 273-286. [CrossRef]

37. Huebsch, N.; Loskill, P.; Mandegar, M.A.; Marks, N.C.; Sheehan, A.S.; Ma, Z.; Mathur, A.; Nguyen, T.N.; Yoo, J.C.; Judge, L.M.; et al. Automated Video-Based Analysis of Contractility and Calcium Flux in Human-Induced Pluripotent Stem Cell-Derived Cardiomyocytes Cultured over Different Spatial Scales. Tissue Eng. Part C Methods 2015, 21, 467-479. [CrossRef] [PubMed]

38. Lane, J.C.E.; Weaver, J.; Kostka, K.; Duarte-Salles, T.; Abrahao, M.T.F.; Alghoul, H.; Alser, O.; Alshammari, T.M.; Biedermann, P.; Burn, E.; et al. Safety of hydroxychloroquine, alone and in combination with azithromycin, in light of rapid wide-spread use for COVID-19: A multinational, network cohort and self-controlled case series study. medRxiv 2020, 2020.2004.2008.20054551. [CrossRef]

39. King, J.H.; Huang, C.L.; Fraser, J.A. Determinants of myocardial conduction velocity: Implications for arrhythmogenesis. Front. Physiol. 2013, 4, 154. [CrossRef] 
40. Khuroo, M.S. Chloroquine and hydroxychloroquine in coronavirus disease 2019 (COVID-19). Facts, fiction and the hype: A critical appraisal. Int. J. Antimicrob. Agents 2020, 56, 106101. [CrossRef]

41. Kitani, T.; Ong, S.G.; Lam, C.K.; Rhee, J.W.; Zhang, J.Z.; Oikonomopoulos, A.; Ma, N.; Tian, L.; Lee, J.; Telli, M.L.; et al. HumanInduced Pluripotent Stem Cell Model of Trastuzumab-Induced Cardiac Dysfunction in Patients With Breast Cancer. Circulation 2019, 139, 2451-2465. [CrossRef]

42. Rosenberg, E.S.; Dufort, E.M.; Udo, T.; Wilberschied, L.A.; Kumar, J.; Tesoriero, J.; Weinberg, P.; Kirkwood, J.; Muse, A.; DeHovitz, J.; et al. Association of Treatment With Hydroxychloroquine or Azithromycin With In-Hospital Mortality in Patients With COVID-19 in New York State. JAMA 2020, 323, 2493-2502. [CrossRef] [PubMed]

43. Takeda, A.; Takano, N.; Kokuba, H.; Hino, H.; Moriya, S.; Abe, A.; Hiramoto, M.; Tsukahara, K.; Miyazawa, K. Macrolide antibiotics enhance the antitumor effect of lansoprazole resulting in lysosomal membrane permeabilizationassociated cell death. Int. J. Oncol. 2020, 57, 1280-1292. [CrossRef] [PubMed]

44. Mukai, S.; Moriya, S.; Hiramoto, M.; Kazama, H.; Kokuba, H.; Che, X.F.; Yokoyama, T.; Sakamoto, S.; Sugawara, A.; Sunazuka, T.; et al. Macrolides sensitize EGFR-TKI-induced non-apoptotic cell death via blocking autophagy flux in pancreatic cancer cell lines. Int. J. Oncol. 2016, 48, 45-54. [CrossRef]

45. Norinder, U.; Tuck, A.; Norgren, K.; Munic Kos, V. Existing highly accumulating lysosomotropic drugs with potential for repurposing to target COVID-19. Biomed. Pharmacother. 2020, 130, 110582. [CrossRef] [PubMed]

46. Scherrmann, J.M. Intracellular ABCB1 as a Possible Mechanism to Explain the Synergistic Effect of HydroxychloroquineAzithromycin Combination in COVID-19 Therapy. AAPS J. 2020, 22, 86. [CrossRef]

47. Cyganek, L.; Tiburcy, M.; Sekeres, K.; Gerstenberg, K.; Bohnenberger, H.; Lenz, C.; Henze, S.; Stauske, M.; Salinas, G.; Zimmermann, W.H.; et al. Deep phenotyping of human induced pluripotent stem cell-derived atrial and ventricular cardiomyocytes. JCI Insight 2018, 3, e99941. [CrossRef]

48. Feyen, D.A.M.; McKeithan, W.L.; Bruyneel, A.A.N.; Spiering, S.; Hormann, L.; Ulmer, B.; Zhang, H.; Briganti, F.; Schweizer, M.; Hegyi, B.; et al. Metabolic Maturation Media Improve Physiological Function of Human iPSC-Derived Cardiomyocytes. Cell Rep. 2020, 32, 107925. [CrossRef]

49. Laing, J.G.; Tadros, P.N.; Green, K.; Saffitz, J.E.; Beyer, E.C. Proteolysis of connexin43-containing gap junctions in normal and heat-stressed cardiac myocytes. Cardiovasc. Res. 1998, 38, 711-718. [CrossRef]

50. Mauthe, M.; Orhon, I.; Rocchi, C.; Zhou, X.; Luhr, M.; Hijlkema, K.J.; Coppes, R.P.; Engedal, N.; Mari, M.; Reggiori, F. Chloroquine inhibits autophagic flux by decreasing autophagosome-lysosome fusion. Autophagy 2018, 14, 1435-1455. [CrossRef]

51. Streckfuss-Bomeke, K.; Wolf, F.; Azizian, A.; Stauske, M.; Tiburcy, M.; Wagner, S.; Hubscher, D.; Dressel, R.; Chen, S.; Jende, J.; et al Comparative study of human-induced pluripotent stem cells derived from bone marrow cells, hair keratinocytes, and skin fibroblasts. Eur. Heart J. 2013, 34, 2618-2629. [CrossRef] [PubMed]

52. Rossler, U.; Hennig, A.F.; Stelzer, N.; Bose, S.; Kopp, J.; Soe, K.; Cyganek, L.; Zifarelli, G.; Ali, S.; von der Hagen, M.; et al. Efficient generation of osteoclasts from human induced pluripotent stem cells and functional investigations of lethal CLCN7-related osteopetrosis. J. Bone Miner. Res. 2021, 36, 1621-1635. [CrossRef] [PubMed]

53. Li, W.; Luo, X.; Ulbricht, Y.; Wagner, M.; Piorkowski, C.; El-Armouche, A.; Guan, K. Establishment of an automated patch-clamp platform for electrophysiological and pharmacological evaluation of hiPSC-CMs. Stem Cell Res. 2019, 41, 101662. [CrossRef]

54. Luo, X.; Li, W.; Kunzel, K.; Henze, S.; Cyganek, L.; Strano, A.; Poetsch, M.S.; Schubert, M.; Guan, K. IP3R-Mediated Compensatory Mechanism for Calcium Handling in Human Induced Pluripotent Stem Cell-Derived Cardiomyocytes With Cardiac Ryanodine Receptor Deficiency. Front. Cell Dev. Biol. 2020, 8, 772. [CrossRef] [PubMed]

55. QuoData. Quality \& Statistics GmbH. Available online: https:/ / quodata.de/ (accessed on 19 August 2021).

56. McQuin, C.; Goodman, A.; Chernyshev, V.; Kamentsky, L.; Cimini, B.A.; Karhohs, K.W.; Doan, M.; Ding, L.; Rafelski, S.M.; Thirstrup, D.; et al. CellProfiler 3.0: Next-generation image processing for biology. PLoS Biol. 2018, 16, e2005970. [CrossRef]

57. Schindelin, J.; Arganda-Carreras, I.; Frise, E.; Kaynig, V.; Longair, M.; Pietzsch, T.; Preibisch, S.; Rueden, C.; Saalfeld, S.; Schmid, B.; et al. Fiji: An open-source platform for biological-image analysis. Nat. Methods 2012, 9, 676-682. [CrossRef]

58. Li, W.; Stauske, M.; Luo, X.; Wagner, S.; Vollrath, M.; Mehnert, C.S.; Schubert, M.; Cyganek, L.; Chen, S.; Hasheminasab, S.M.; et al. Disease Phenotypes and Mechanisms of iPSC-Derived Cardiomyocytes From Brugada Syndrome Patients With a Loss-of-Function SCN5A Mutation. Front. Cell Dev. Biol. 2020, 8, 592893. [CrossRef]

59. Li, W.; Luo, X.; Ulbricht, Y.; Guan, K. Blebbistatin protects iPSC-CMs from hypercontraction and facilitates automated patch-clamp based electrophysiological study. Stem Cell Res. 2021, 56, 102565. [CrossRef] 